\title{
Lorentzian vacuum transitions: Open or closed universes?
}

\author{
Sebastián Céspedes $\odot,{ }^{1}$ Senarath P. de Alwis, ${ }^{2}$ Francesco Muia, ${ }^{3}$ and Fernando Quevedo ${ }^{3}$ \\ ${ }^{1}$ Instituto de Física Teórica UAM-CSIC C/ Nicolás Cabrera 13-15, \\ Campus de Cantoblanco, 28049 Madrid, Spain \\ ${ }^{2}$ Physics Department, University of Colorado, Boulder, Colorado 80309, USA \\ ${ }^{3}$ DAMTP, Centre for Mathematical Sciences, Wilberforce Road, Cambridge CB3 OWA, United Kingdom
}

(Received 12 December 2020; accepted 8 June 2021; published 26 July 2021)

\begin{abstract}
We consider the generalization of quantum tunneling transitions in the WKB approximation to the timeindependent functional Schrödinger and Wheeler-DeWitt equations. Following a Lorentzian approach, we compute the transition rates among different scalar field vacua and compare with those performed by Coleman and collaborators using the Euclidean approach. For gravity, we develop a general formalism for computing transition rates in Wheeler's superspace. This is then applied to computing decays in flat space and then to transitions in the presence of gravity. In the latter case we point out the complexities arising from having nonpositive definite kinetic terms illustrating them in the simplified context of minisuperspace. This corresponds to a generalization of the well-known 'tunneling from nothing' scenarios. While we can obtain the leading term for the transitions obtained by Euclidean methods we also point out some differences and ambiguities. We show that there is no obstruction to keeping the spherically $(S O(4))$ symmetric closed slicing for the new vacuum after a de Sitter to de Sitter transition. We argue that this is the natural Lorentzian realization of the Coleman-De Luccia instanton and that a closed universe is also obtained if the mini-superspace assumption is relaxed. This is contrary to the open universe predicted by Coleman-De Luccia which relies on an analytic continuation performed after bubble nucleation. Our findings may have important cosmological implications related to the origin of inflation and to the string landscape. In particular, they question the widespread belief that evidence for a closed universe would rule out the string landscape.
\end{abstract}

DOI: $10.1103 /$ PhysRevD.104.026013

\section{INTRODUCTION}

Vacuum decay may be the cause of both the beginning and end of our universe. It is also one of the very few systems in which the quantum aspects of gravity are crucial in order to have a proper description of the physical process. Euclidean methods, developed mostly by Coleman and collaborators [1-3], have been used to extend the well understood WKB quantum mechanics techniques to field theory and gravity. Decay rates have been computed for simple systems, corresponding to scalar field potentials with several minima, mostly in the thin-wall approximation. Related investigations by Brown and Teitelboim involved studying the nucleation of branes interpolating between vacua of different values of the cosmological constant [4]. However, while the quantum mechanical calculations are well under control, the extensions to field theory and gravity rely on

Published by the American Physical Society under the terms of the Creative Commons Attribution 4.0 International license. Further distribution of this work must maintain attribution to the author(s) and the published article's title, journal citation, and DOI. Funded by SCOAP. extrapolations such as analytic continuations and approximations, such as dilute instantons, that are not fully justified especially in the presence of gravity (for a review see for instance [5], and for a recent comprehensive and critical discussion see [6]).

A Hamiltonian approach to vacuum transitions that describes them directly without the need to use Euclidean techniques was developed by Fischler, Morgan and Polchinski (FMP) [7,8]. Solving the Hamiltonian constraints and the Israel matching conditions for the system of two spacetimes with different cosmological constants, together with the bubble wall (brane) separating them, allowed them to compute the transitions from a Schwarzschild black hole (i.e., spherically symmetric asymptotically Minkowski) spacetime to a de Sitter (dS) spacetime, but the formalism applies to all vacuum transitions considered by Coleman-De Luccia (CDL). The motivation for their calculation was the series of papers by Guth and collaborators on the theme of creating an inflating 'universe in the lab' culminating in work of Farhi, Guth and Guven (FGG) [9]. The latter was based on a Euclidean instanton construction whose validity was somewhat questionable (as pointed out by the authors of the paper 
themselves). The reason was that this instanton was singular and the question of whether it should be included in the Euclidean functional integral became an issue. However the fact that the Hamiltonian calculation of the decay rate agrees with the Euclidean approach, implied (as FMP argued) that the final results for the transition rates are robust despite the fact that Euclidean singular instanton calculation was not well defined.

One important difference between the Euclidean and Hamiltonian approaches is the fact that in the Euclidean approach a series of analytic continuations (which go beyond what may be justified by WKB quantum mechanics) are needed in order to find the Lorentzian geometry after the transition. Even though the starting point may be a closed universe with $S O(4)$ spherical symmetry, the resulting geometry (inside the light cone of an observer at the center of the nucleated bubble) after the transition turns out to correspond to an open universe with hyperbolic symmetry. However in the Hamiltonian approach of FMP there is no such implication. The entire analysis is done within the context of a spherically symmetric $[S O(3)]$ ansatz but the calculation does not imply the CDL argument for an open universe. ${ }^{1}$ Furthermore the natural Lorentzian mini-superspace calculation corresponding to the CDL Euclidean calculation would have $S O(4)$ symmetry and hence necessarily gives rise to a closed universe as we discuss later. This is an important difference especially if we consider the possibility that our own universe could be the result of a vacuum transition. Actually, based on the CDL result, it has been claimed that this is the only generic prediction of the string landscape $[10,11] .^{2}$

To the best of our knowledge, the fact that the Hamiltonian formalism developed by FMP can give rise to a closed universe has not been emphasized so far. Probably because the approach of FMP was originally developed in order to address the question of Minkowski black hole to dS transition that was posed by [9] in the Euclidean approach. However, the FMP formalism applies also to all other potential vacuum transitions (dS to dS, Minkowski to dS as well as transitions involving antide Sitter (AdS) [14-18]). One limitation of this approach is that it only describes transitions among two spacetimes differing by the vacuum energy separated by a brane, whereas in general the CDL approach is formulated in

\footnotetext{
${ }^{1}$ As is well known, dS space allows several foliations including those that correspond to closed, open and flat slicings. In CDL the symmetries of the scalar field determine the preferred slicing after analytic continuation. However the latter cannot be justified by standard WKB arguments and this argument is not meaningful in the Hamiltonian formalism.

${ }^{2}$ For a different view based on the "no boundary wave function", see the extensive work of Hawking, Hartle and Hertog (for example [12] and references therein). In particular in [13] it has been argued based on a dS/CFT conjecture that the probability of observing negative curvature on exit from eternal inflation is exponentially suppressed.
}

terms of scalar potentials with different minima and barriers between them. However, in actual practice most such calculations reverted to the thin-wall approximation so that there was no essential difference to having the spaces separated by a brane as in the Brown-Teitelboim [4] (BT) calculation. Furthermore the landscape of string theory results from transitions due to the nucleation of branes, which in the effective field theory approach are of string scale thickness, and hence effectively a thin wall so, as discussed by Bousso and Polchinski [19] for instance, the $\mathrm{BT}$ process (and hence FGG/FMP) is more relevant for the landscape of string theory than the scalar field process of CDL. The latter is more relevant for questions of eternal inflation and other transitions such as the transition towards decompactification, however.

A generalization of the FMP formalism to include explicit scalar field potentials is still an open question. Here, we are only partially successful in addressing this since we do it only in a mini-superspace model in which the metric and scalar field only depend on time. Nevertheless we find several interesting results essentially extending the 'tunneling from nothing' arguments of Hartle-Hawking, Vilenkin and Linde [20-23], and then compare with the Euclidean approach. ${ }^{3}$ We start by revisiting the extension of the WKB approximation to field theory by solving the functional, time-independent Schrödinger equation in the WKB approximation. We reproduce the Euclidean results for the (exponential term in the) decay rates but within a totally different method. The prefactor however is different and there is no problem with negative modes. ${ }^{4}$ Next we include gravity albeit in a mini-superspace model and then we discuss the extension (for the case of a brane) to an $S O(3)$ symmetric situation following FMP and our earlier work. One of our concrete results is to confirm the fact that in the Hamiltonian approach, the end result for the geometry of the remaining universe may be a closed rather than an open universe. We then study the physical implications of this result comparing with previous studies of CDL transitions.

The article is organized as follows:

(a) In Sec. II we develop the formalism to address quantum transitions in Wheeler's superspace adapting the semiclassical WKB approximation in a covariant superspace approach. Given the nature of the Hamiltonian constraint leading to the Wheeler-DeWitt equation [25], the corresponding Schrödinger wave functional is time independent. The transition probabilities are ratios of squares of wave functionals for the different configurations. Expanding on standard WKB techniques in quantum mechanics and on previous

\footnotetext{
${ }^{3}$ Of course in the context of the "no boundary wave function" Hawking and collaborators have long advocated for a landscape of closed universes. See for example [12] and references therein.

${ }^{4}$ For a recent discussion of the negative modes issues see for instance [24] and references therein.
} 
approaches towards field theory [26-30], we find general expressions, covariant on a generalized Wheeler superspace, for the leading and next order corrections to the wave functions. In particular, we find a general closed expression for the semiclassical wave functional with the prefactor given by the analog of the Van Vleck determinant.

(b) In Sec. III we apply the formalism of Sec. II to the flat space field theoretical case of a scalar field potential neglecting the effects of gravity. In particular, following the textbook quantum mechanics matching conditions we explicitly compute the $S$-matrix as well as the lifetime of the corresponding resonances and obtain the decay rate. In contrast to the calculations of Coleman and collaborators [1-3], in our calculation we find that there is no issue with negative modes or analytic continuation of a manifestly real amplitude to get an imaginary part to the energy that can be interpreted as a decay width. Instead in our calculation the decay width is identified in the standard way as the imaginary part of a complex pole in the $S$-matrix.

(c) In Sec. IV we include gravity but in order to have explicit results we concentrate on the mini-superspace model in order to compare with the CDL Euclidean approach. In this case superspace reduces to a twodimensional space with coordinates the scalar field $\phi(t)$ and the metric scale factor $a(t)$. We find a general expression for the decay rate which in the thin-wall approximation gives exactly the CDL result but with an unclear interpretation. Furthermore, the fact that the metric in superspace is not positive definite allows for classical paths to connect the two dS minima without the need to pass through the barrier modifying substantially the results for the transition rate as compared with CDL. We argue that both approaches may be addressing different questions. We emphasize the difference between the two approaches. Even though the calculations in both cases can be said to be done in mini-superspace with an $S O(4)$ symmetry, in CDL, after analytic continuation the standard picture of vacuum transition with a wall separating the two dS spacetimes emerges turning the original $S O(4)$ symmetry into $S O(3,1)$. Whereas in the Lorentzian approach the $S O(4)$ symmetry remains. We also point out the main difference; the fact that CDL implies an open universe whereas we, as with the 'tunneling from nothing' scenarios and FMP, find a closed universe. Finally we briefly discuss the possibility of purely classical transitions from one local minimum to another through an initially contracting phase.

(d) In Sec. $\mathrm{V}$ we quickly review the relevant points of FMP to extend the results of the previous sections beyond mini-superspace, though with the restriction that the matter sector includes only the two cosmological constants and no scalar field. We describe the trajectory of the wall after nucleation and find it similar to CDL with the curious fact that the speed of the wall reaches a maximum which is less than the speed of light. Then we review the CDL arguments to obtain an open universe and revise the other implications for early universe cosmology as addressed for instance in [10]: impact on CMB, the number of inflation $e$-folds, etc. We then concentrate on the implications of having a closed rather than an open universe after the transition. We point out the physical differences not only regarding the potential for measuring the curvature of the universe, but also on how inflation is obtained after the transition, address the constrains on the number of $e$-foldings and the effect on density perturbations.

(e) Finally we discuss open questions and give a general outlook in the concluding section.

\section{WKB FOR FIELD THEORY AND QUANTUM GRAVITY}

In this section we develop a general formalism to generalize the WKB formalism for vacuum decay to the Wheeler-DeWitt (WDW) wave function $\Psi$ in Wheeler's superspace. The Wheeler-DeWitt equation [25] is a constraint equation on the space of wave functionals that describe a gravitational system. The ratios of absolute squares of the WDW wave functionals for the different configurations $\mathcal{M}_{1}$ and $\mathcal{M}_{2}$ can then be interpreted as relative probabilities for realizing them $[31,32]$.

This interpretation is still under debate. An alternative interpretation is to consider a fixed gravitational background-such as the FRW background-and regard the WDW equation as a Klein-Gordon equation and interpret the timelike component of the probability current as the probability density [25] (see also [33,34]). However this results in a nonpositive probability density as observed in [25] unless one picks just the expanding phase. Also this is necessarily an introduction of time within the limited context of a given gravitational background ignoring the back reaction of matter.

Many of the calculations done in the field of quantum cosmology (including some of the calculations below) are in fact done in mini-superspace where one might ask whether it is not appropriate to use the Klein-Gordon approach to incorporating a notion of time as for instance in the proposal of [25] and adopted by [35] (see Sec. IV). This involves (for the case of a scalar field $\phi$ in minisuperspace with a scale factor $a$ ) factoring the WDW wave function as $\Psi(a, \phi)=\psi(a) \chi(a, \phi)$. One then picks for $\psi$, which in general is a superposition of the expanding and contracting phase, the purely expanding component. If this is done then the time component of the probability current would be positive definite and then $\chi$ would have the standard quantum mechanical interpretation of the probability of finding the value $\phi$ at the 'time' $a$. 
However this interpretation will only work for Vilenkin's 'tunneling wave function' - where the appropriate boundary condition of an expanding universe in the classical region is imposed. It will not be appropriate for the more general case and, in particular, not for the Hartle-Hawking wave function. Furthermore, it begs the question "what is the meaning of $\psi(a)$ ?" We prefer therefore to follow the interpretation originally given in [31,32] and widely followed by Hawking and collaborators, and other workers in the field of quantum cosmology, over the past 35 years. In particular, in discussing the relative probability of nucleating an entire universe we believe that this interpretation is more appropriate since one looks for a solution of the coupled gravity matter system in the WKB approximation but without fixing oneself to a given gravitational background. In other words one is at least in principle taking into account the backreaction of matter even though the quantum corrections are computed to leading order in $\hbar$.

In order to be completely general (and in particular not having to choose between Vilenkin's proposal and that of Hartle and Hawking for the wave function of the universe) we adhere to the interpretation of the WDW wave function followed by $[31,32]$ and which we use in the form given in the following equation:

$$
\mathcal{P}\left(\mathcal{M}_{1} \rightarrow \mathcal{M}_{2}\right)=\frac{\left|\Psi\left(\mathcal{M}_{2}\right)\right|^{2}}{\left|\Psi\left(\mathcal{M}_{1}\right)\right|^{2}}
$$

In connection with selecting an expanding branch for background gravity, in order to define time in the sense of Schrödinger evolution, it also behooves us to mention two other early papers. In the work of Hajicek [36] it was pointed out, using the functional integral calculation, that the Feynman kernel for quantum gravity, as a result of integrating over the lapse, is in fact a projector onto the physical states (i.e., states satisfying the constraint). Also in this paper the Hartle-Hawking wave function is recalculated and the fact that this has no definite direction of time (being a superposition of expanding and contracting phases of the de Sitter spacetime) was pointed out. Further remarks in this direction have been made by Zeh [37] who showed that the time parameter introduced via an equation of the form $\partial_{t}=\nabla S \cdot \nabla$ with $S$ being the classical action), has no absolute meaning in the absence in the wave function of a factor $e^{i E t}$ referring to absolute time. ${ }^{5}$

The discussion in the next subsection may be trivially specialized to the case of (flat space) field theory in which case the (spatial integral of the) WDW equation is the time independent Schrödinger equation.

\footnotetext{
${ }^{5}$ We thank an anonymous referee for pointing out these arguments and for these and several other references to the early literature on these subjects.
}

\section{A. Semiclassical expansion for the WDW equation}

The WKB approximation for the WDW equation has been considered by many authors beginning with the original work of DeWitt [25] (see also [33,34]).

We assume that spacetime can be foliated into a family of nonintersecting spacelike three-slices that can be seen (at least locally) as the level surfaces of a scalar function $t$. The function $t$ can be interpreted as a global time function. Given a line element of the generic form

$d s^{2}=\left(-N_{t}^{2}+N_{i} N^{i}\right) d t^{2}+2 N_{i} d t d x^{i}+\gamma_{i j} d x^{i} d x^{j}$,

where $N_{t}, N_{i}$ are lapse and shift, while $\gamma_{i j}$ is the spatial three-dimensional metric, the gravitational contribution to the Lagrangian of the system can be written as

$$
L_{g}=\int d^{3} x N_{t} \sqrt{\gamma}\left(K_{i j} K^{i j}-K^{2}+{ }^{(3)} R\right),
$$

where $K_{i j}=\frac{1}{2 N_{t}}\left(\partial_{i} N_{j}+\partial_{j} N_{i}-\partial_{0} \gamma_{i j}\right)$ is the extrinsic curvature, $K=\gamma^{i j} K_{i j}$ and ${ }^{(3)} R$ is the intrinsic curvature of the three-dimensional slice. We denote the canonically conjugate momentum to a field $\Phi^{M}$ by $\pi_{M}$. The primary constraints of the system arising from Eq. (2.3) are $\pi_{N_{t}} \approx 0, \pi_{N_{i}} \approx 0$, where $\approx$ means that they are constraints on the classical solutions. In the quantum case we correspondingly have constraints on the space of wave functionals $\Psi(\Phi)$, where $\Phi$ collectively denotes the three-metric and matter fields (and their spatial derivatives up to second order) present in the system. Note that the classical constraints $\pi_{N_{t}} \approx 0, \pi_{N_{i}} \approx 0$ imply that the wave function $\Psi$ is independent of $N_{t}, N_{r}$. The full system is described by $L_{g}+L_{\text {mat }}$ where $L_{\text {mat }}$ is the Lagrangian that describes the matter present in the system.

The Hamiltonian constraint takes the general form

$$
\mathcal{H}=\frac{1}{2} G^{M N}(\Phi) \pi_{M} \pi_{N}+f[\Phi] \approx 0
$$

where $G_{M N}$ is the metric on the $d$-dimensional field space, including all the components of $\gamma_{i j}$ and the matter fields, and can be read from the kinetic terms of $L_{g}+L_{\text {mat }}$. At the same time, the momentum constraint $\mathcal{P}_{i} \approx 0$ has to hold. Up to operator ordering ambiguities which are fixed by demanding the derivatives with respect to the components $\Phi^{M}$ of $\Phi$ are covariant with respect to the metric $G_{M N}$ (which we emphasize is not positive definite in the presence of gravity), we have for the WDW equation (replacing $\left.\pi_{M} \rightarrow-i \hbar \nabla_{M}\right)$

$\mathcal{H} \Psi(\Phi)=\left[-\frac{\hbar^{2}}{2} G^{M N}(\Phi) \nabla_{M} \nabla_{N}+f[\Phi]\right] \Psi(\Phi)=0$.

As usual we can write 


$$
\Psi[\Phi]=e^{\frac{i}{\hbar} S[\Phi]},
$$

and define the semiclassical expansion

$$
S[\Phi]=S_{0}[\Phi]+\hbar S_{1}[\Phi]+O\left(\hbar^{2}\right) .
$$

Substituting Eq. (2.6) and Eq. (2.7) in the WDW equation we can in principle determine recursively the semiclassical expansion coefficients. The lowest two orders give

$$
\begin{gathered}
\frac{1}{2} G^{M N} \frac{\delta S_{0}}{\delta \Phi^{M}} \frac{\delta S_{0}}{\delta \Phi^{N}}+f[\Phi]=0, \\
2 G^{M N} \frac{\delta S_{0}}{\delta \Phi^{M}} \frac{\delta S_{1}}{\delta \Phi^{N}}=i G^{M N} \nabla_{M} \nabla_{N} S_{0} .
\end{gathered}
$$

In Hamilton-Jacobi theory, which corresponds to the classical limit of the quantum calculation, $\pi_{M}=\frac{\partial S}{\partial \Phi^{M}}$. Observe that at a turning point $\pi_{M}=\frac{\delta S_{0}}{\delta \Phi^{M}}=0$ for all $M$, and the semiclassical expansion breaks down since $S_{1}$ cannot be determined.

Let us now introduce on the selected spatial slice a set of integral curves, parametrized by $s$, on the field manifold

$$
C(s) \frac{d \Phi^{N}}{d s}=G^{M N} \frac{\delta S_{0}}{\delta \Phi^{M}} .
$$

Given the constraints, the classical action (on a classical trajectory) becomes

$$
\begin{aligned}
S_{0}\left[\Phi_{s}\right] & =\int^{\Phi_{s}} \int_{X} \pi_{M} d \Phi^{M} \\
& =\int^{s} d s^{\prime} \int_{X} \frac{\delta S_{0}}{\delta \Phi^{M}} \frac{d \Phi^{M}}{d s^{\prime}} \\
& =\int^{s} d s^{\prime} C^{-1}\left(s^{\prime}\right) \int_{X} \frac{\delta S_{0}}{\delta \Phi^{M}} G^{M N} \frac{\delta S_{0}}{\delta \Phi^{N}} \\
& =-2 \int^{s} d s^{\prime} C^{-1}\left(s^{\prime}\right) \int_{X} f\left[\Phi_{s^{\prime}}\right] .
\end{aligned}
$$

We have used Eq. (2.10) in the third line and Eq. (2.8) in the fourth. Similarly from Eq. (2.8) after integrating over the spatial slice $X$ we have

$$
\frac{d S_{1}}{d s}=\int_{X} \frac{d \Phi^{N}}{d s} \frac{\delta S_{1}}{\delta \Phi^{N}}=\frac{i}{2} C^{-1}(s) \int_{X} \nabla^{2} S_{0}
$$

giving

$$
S_{1}\left[\Phi_{s}\right]=\frac{i}{2} \int^{s} d s^{\prime} C^{-1}\left(s^{\prime}\right) \int_{X} \nabla^{2} S_{0}\left[\Phi_{s^{\prime}}\right] .
$$

For a given parametrization $C(s)$ one can in principle solve the first order differential equation in Eq. (2.10) [with $S_{0}$ given by Eq. (2.11) to get $\Phi_{s}$ as a function of $s$ and substituting in Eq. (2.13) the semiclassical correction $S_{1}$ is determined away from any turning points (caustics)].

One can also choose a parameter $\tau$ to be the distance function along the trajectories, defined as

$$
d \tau^{2} \equiv \int_{X} \delta \Phi^{M} G_{M N} \delta \Phi^{N}
$$

we get using Eq. (2.10) and Eq. (2.8)

$$
\left(\frac{d \tau}{d s}\right)^{2}=\int_{X} \frac{\delta \Phi^{M}}{d s} G_{M N} \frac{\delta \Phi^{N}}{d s}=-2 C^{-2}(s) \int_{X} f[\Phi] .
$$

Solving this for $C(s)$ we have from Eq. (2.11) for the classical action $S_{0}$ (with an arbitrary parametrization of the integration trajectory)

$$
\begin{aligned}
S_{0}\left[\Phi_{s}\right]-S_{0}\left[\Phi_{0}\right]= & \int_{0}^{s} d s^{\prime}\left[\int_{X} \frac{d \Phi_{s^{\prime}}^{M}}{d s^{\prime}} G_{M N} \frac{d \Phi_{s^{\prime}}^{N}}{d s^{\prime}}\right]^{1 / 2} \\
& \times\left[\int_{X}\left(-2 f\left[\Phi_{s^{\prime}}\right]\right]^{1 / 2} .\right.
\end{aligned}
$$

The classical path along which this has to be evaluated is of course the one which extremizes this action with the end points fixed. The variational derivative may be worked out easily by observing that the variation of the first factor gives the left-hand side of the geodesic equation on superspace. Introducing the metric compatible connection on superspace (Vilkovsky connection) $D / D \tau=\frac{d \Phi^{M}}{d \tau} \nabla_{M}$ we get

$$
G_{P N} \Sigma\left[\Phi_{\tau}\right] \frac{D}{D \tau}\left(\Sigma\left[\Phi_{\tau}\right] \frac{d \Phi_{\tau}^{N}}{d \tau}\right)+\frac{\delta f\left[\Phi_{\tau}\right]}{\delta \Phi^{P}}=0
$$

where after doing the variation we have set $s=\tau$ and defined $\Sigma\left[\Phi_{\tau}\right] \equiv \sqrt{-2 \int_{X} f\left[\Phi_{\tau}\right]}$. Or defining the affine parameter $\sigma$ by $d \sigma=d \tau / \Sigma\left[\Phi_{\tau}\right]$ [note that this corresponds to $s$ if we choose $C(s)=1$ in Eq. (2.15)] we have the classical equations of motion [that would follow also directly from the Hamiltonian in Eq. (2.4)]

$$
\frac{D}{D \sigma} \frac{d \Phi_{\sigma}^{N}}{d \sigma}+G^{N P} \frac{\delta f\left[\Phi_{\sigma}\right]}{\delta \Phi^{P}}=0
$$

We remark in passing that this equation of motion does not have an obvious interpretation as Lorentzian or Euclidean since in the presence of gravity (as we stressed before) the superspace metric $G_{M N}$ is not positive definite. We will see the consequences of this explicitly when we discuss the minisuperspace example. Now going back to Eq. (2.15) and putting $s=\tau$,

$$
C^{2}(\tau)=-2 \int_{X} f[\Phi]
$$


Hence in terms of $\tau$ we have

$$
\begin{aligned}
S_{0}\left[\Phi_{\tau}\right] & =\int^{\tau} d \tau^{\prime} \sqrt{-2 \int_{X} f\left[\Phi_{\tau^{\prime}}\right] .} \\
S_{1}\left[\Phi_{\tau}\right] & =\frac{i}{2} \int^{\tau} d \tau^{\prime} \frac{1}{\sqrt{-2 \int_{X} f\left[\Phi_{\tau^{\prime}}\right]}} \int_{X} \nabla^{2} S_{0}\left[\Phi_{\tau^{\prime}}\right],
\end{aligned}
$$

\section{B. Wave function and van Vleck determinant}

In the corresponding multidimensional quantum mechanical case the expression for $S_{1}$ is given by the VanVleck determinant [38] (see also [39]). To see the connection we first observe (essentially generalizing an argument in [30]) the following:

Changing to the coordinates defined with respect to the orthonormal basis we have for a variation around a trajectory

$$
\delta \Phi^{M}(x)=\delta \tau t^{M}(x)+\delta \lambda^{\bar{P}} \frac{\partial \Phi^{M}}{\partial \lambda^{\bar{P}}},
$$

where $t^{M}=\partial \Phi^{M} / \partial \tau$, while

$$
\frac{\partial}{\partial \lambda^{\bar{P}}}=\int_{X} \frac{\partial \Phi^{M}}{\partial \lambda^{\bar{P}}} \frac{\partial}{\partial \Phi^{M}} .
$$

The vectors defined in Eq. (2.22) are orthogonal to the vector $\partial / \partial \tau=\int_{X}\left(\partial \Phi^{M} / \partial \tau\right) \partial / \partial \Phi^{M}$.

Let us denote the components in the original coordinates (including the spatial position $x$ which is to be treated as an index as $A=\{M, x\}$ ). In other words it is convenient to use DeWitt's condensed notation treating the set of fields (including metric components) $\left\{\Phi^{M}\right\}$ as a set of "coordinates" i.e., $q^{i} \rightarrow \Phi^{M x}$ where the spatial variable $x$ is treated as an index with the understanding that sums over $x$ are integrals and Kronecker deltas are replaced by Dirac delta functions. The superspace metric in the new coordinate system $\left(\bar{A}=\tau, \lambda^{\bar{P}}\right)$ is

$$
\bar{G}_{\bar{A} \bar{B}}=\frac{\partial \Phi^{A}}{\partial \lambda^{\bar{A}}} G_{A B} \frac{\partial \Phi^{B}}{\partial \lambda^{\bar{B}}},
$$

therefore

$$
\begin{aligned}
G^{A B} \nabla_{A} \nabla_{B} S_{0} & =G^{\bar{A} \bar{B}} \nabla_{\bar{A}} \nabla_{\bar{B}} S_{0}=\frac{1}{\sqrt{\bar{G}}} \partial_{\bar{A}}\left(\sqrt{\bar{G}} G^{\bar{A} \bar{B}} \partial_{\bar{B}} S_{0}\right) \\
& =\frac{1}{\sqrt{\bar{G}}} \partial_{\bar{A}}\left(\frac{\partial \lambda^{\bar{A}}}{\partial \Phi^{A}} \sqrt{\bar{G}} G^{A B} \partial_{B} S_{0}\right) \\
& =\frac{1}{\sqrt{\bar{G}}} \partial_{\bar{A}}\left(\frac{\partial \lambda^{\bar{A}}}{\partial \Phi^{A}} \sqrt{\bar{G}} C(\tau) \frac{\partial \Phi^{A}}{\partial \tau}\right) \\
& =\frac{1}{\sqrt{\bar{G}}} \partial_{\bar{A}}\left(\sqrt{\bar{G}} C(\tau) \frac{\partial \lambda^{\bar{A}}}{\partial \tau}\right) \\
& =\frac{1}{\sqrt{\bar{G}}} \partial_{\tau}(\sqrt{\bar{G}} C(\tau))
\end{aligned}
$$

In the second line we used Eq. (2.10). From Eq. (2.12) we have

$$
\begin{aligned}
\frac{d S_{1}}{d \tau} & =\frac{i}{2} \partial_{\tau} \ln (C(\tau) \sqrt{\bar{G}}) \\
& =\frac{i}{2} \partial_{\tau} \ln \left(C(\tau) \operatorname{det} \frac{\partial \Phi^{A}}{\partial \lambda^{\bar{A}}} \sqrt{G}\right) .
\end{aligned}
$$

Noting that the line element on superspace may be rewritten as

$$
\begin{aligned}
d s^{2} & =\bar{G}_{\bar{A} \bar{B}} d \lambda^{\bar{A}} d \lambda^{\bar{B}} \\
& =d \tau^{2}+G_{A B} \frac{d \Phi^{A}}{d \lambda^{\bar{N}}} \frac{d \Phi^{B}}{d \lambda^{\bar{M}}} d \lambda^{\bar{N}} d \lambda^{\bar{M}},
\end{aligned}
$$

we have [using also Eq. (2.20)]

$$
\begin{aligned}
S_{1}\left[\Phi_{\tau}\right]= & \frac{i}{2} \ln \sqrt{-2 \int_{X} f\left[\Phi_{\tau}\right]}+\frac{i}{2} \ln \sqrt{\left(\operatorname{det} G_{A B} \frac{d \Phi^{A}}{d \lambda^{\bar{N}}} \frac{d \Phi^{B}}{d \lambda^{\bar{M}}}\right)_{\tau}} \\
& + \text { constant. }
\end{aligned}
$$

Thus the integral in the second term of Eq. (2.20) for $S_{1}$ is in fact the log of the determinant of the superspace metric in the orthogonal directions to the trajectory defined by $\partial / \partial \tau$. Thus the semiclassical wave function may be written as

$$
\begin{aligned}
\Psi\left[\Phi_{\tau}\right]= & \frac{\left[-2 \int_{X} f\left[\Phi_{0}\right]\right]^{1 / 4}}{\left[-2 \int_{X} f\left[\Phi_{\tau}\right]\right]^{1 / 4}} \frac{\left(\operatorname{det} G_{A B} \frac{d \Phi^{A}}{d \lambda^{\tilde{N}}} \frac{d \Phi^{B}}{d \lambda^{\bar{M}}}\right)_{0}^{1 / 4}}{\left(\operatorname{det} G_{A B} \frac{d \Phi^{A}}{d \lambda^{\tilde{N}}} \frac{d \Phi^{B}}{d \lambda^{\bar{M}}}\right)_{\tau}^{1 / 4}} \\
& \times e^{\frac{i}{\hbar}\left(S_{0}\left[\Phi_{\tau}\right]-S_{0}\left[\Phi_{0}\right]\right)} \Psi\left[\Phi_{0}\right],
\end{aligned}
$$

with $S_{0}$ given by Eq. (2.20). This formula generalizes one obtained for many particle quantum mechanics and canonical QFT in [26-28,30].

Alternatively, we can rewrite the expression for this in terms of the (generalization of) the VanVleck determinant. To see this we go back to Eq. (2.12) and choose the parameter $s$ such that $C(s)=1$. Then the calculation in Eq. (2.24) shows that

$$
S_{1}\left[\Phi_{s}\right]-S_{1}\left[\Phi_{0}\right]=\frac{i}{2} \ln \left(\operatorname{det} \frac{\delta \Phi^{A}}{\delta \lambda^{\bar{A}}} \sqrt{G}\right) .
$$

Assuming that the complete integral of the Hamilton-Jacobi equation depends on a set of parameters $\alpha_{\bar{A}}$, we can identify the conjugate variables with our parameters $\lambda^{\bar{A}}$, i.e.,

$$
\lambda^{\bar{A}}=\frac{\delta S_{0}\left[\Phi_{s} ; \alpha\right]}{\delta \alpha^{\bar{A}}} .
$$

The VanVleck matrix can then be written as 


$$
\left[\frac{\delta^{2} S_{0}}{\delta \Phi^{A} \delta \alpha^{\bar{A}}}\right]=\left[\frac{\delta \lambda^{\bar{A}}}{\delta \Phi^{A}}\right]=\left[\frac{\delta \Phi^{A}}{\delta \lambda^{\bar{A}}}\right]^{-1}
$$

Therefore,

$$
S_{1}\left[\Phi_{s}\right]=-\frac{i}{2} \ln \left(\operatorname{det}\left[\frac{\delta^{2} S_{0}}{\delta \Phi^{A} \delta \alpha^{\bar{A}}}\right] \sqrt{G}\right)_{s}+\text { constant. }
$$

Finally, the wave function with semiclassical corrections take the form

$$
\begin{aligned}
\Psi\left[\Phi_{s}\right] & =\frac{1}{P_{0}} \sqrt[4]{G_{s}} \sqrt{\operatorname{det}\left[\frac{\delta^{2} S_{0}}{\delta \Phi^{A} \delta \alpha^{\bar{A}}}\right]_{s}} e^{i \frac{i}{\hbar} S_{0}[\Phi s]} \Psi\left[\Phi_{0}\right], \\
S_{0}\left[\Phi_{s}\right] & =\int_{o}^{s} d s^{\prime} \sqrt{-2 \int_{X} f\left[\Phi_{s^{\prime}}\right] d s^{\prime}}+\text { constant },
\end{aligned}
$$

with the constant $1 / P_{0}$ fixed such that the left-hand side at $s=0$ agrees with the right-hand side at $s=0$.

A formula similar to this in the context of flat space field theory has been given by Bitar and Chang [29]. However the prefactor was obtained there, not by following the WKB method for getting it, but by switching to a functional integral over the fluctuations around the classical path. This gives them a prefactor which is the inverse of the VanVleck prefactor above. Furthermore these authors (in contrast to those of $[28,30])$ claim agreement with the prefactor in the Euclidean instanton analyis of Coleman et al. [1-3]. However we fail to see this. For instance the latter depended on the dilute gas approximation and relied crucially on the presence of a single negative mode in the fluctuations around it. Clearly in the above formula the issue of negative modes do not play a special role. In the next two sections we will elaborate on these differences.

\section{WKB IN FLAT SPACE}

In this section we will recall how to use WKB for the study of vacuum decay in field theory, in flat space. We will analyze two different situations, corresponding to the two potentials in Fig. 1. The left panel corresponds to the process of vacuum decay from a false vacuum to a true vacuum characterized by a lower energy density. In this context, we will show explicitly how the leading order final result corresponds to the CDL bounce, and how the analytic continuation is well justified by the fact that there is an under-the-barrier integral, which is equivalent to analytically continuing time to an imaginary variable. In this case, the WKB formalism can be used to compute the transmission coefficient $T^{2}=\frac{\left|\psi\left(\phi_{B}\right)\right|^{2}}{\left|\psi\left(\phi_{A}\right)\right|^{2}}$, that gives a measure of the decay rate. In the case corresponding to the right panel of Fig. 1 we are able to be more precise; as the potential asymptotically goes to zero, we can define the $S$-matrix for such a system and we will show how to compute the decay rate exactly from the interpretation of a resonance as a complex pole of the $S$-matrix.

Consider the potential in Fig. 1. We need to solve the flat space version of Eq. (2.5). Now we have a global constraint — classically it is a constraint on the Hamiltonian (rather than the density),

$$
H=\int_{X}\left[\frac{\pi_{\phi}^{2}}{2}+\frac{1}{2}\left(\nabla_{x} \phi\right)^{2}+V(\phi)\right]=E,
$$

and hence the Schrödinger equation

$$
\int_{X}\left[-\frac{\hbar^{2}}{2} \frac{\delta^{2}}{\delta \phi(x)^{2}}+\frac{1}{2}\left(\nabla_{x} \phi\right)^{2}+V(\phi)\right] \Psi[\phi]=E \Psi[\phi] .
$$

Using $G^{\phi \phi}=1$ in Eq. (2.8) and Eq. (2.9)

$$
\int_{X} f[\phi]=\int_{X}\left[\frac{1}{2}\left(\nabla_{x} \phi\right)^{2}+V(\phi)\right]-E \equiv U[\phi]-E,
$$

so Eq. (2.11) becomes

$$
S_{0}[\phi]=-2 \int{ }^{s} d s^{\prime} C^{-1}(s)\left[U\left[\phi_{s^{\prime}}\right]-E\right] .
$$

Note that in order to make these expressions well defined we choose the field configuration $\phi$ such that at large $|\mathbf{x}|$ it goes asymptotically to $\phi_{A}$ rapidly enough to make all the integrals above finite. Alternatively as we will do in the
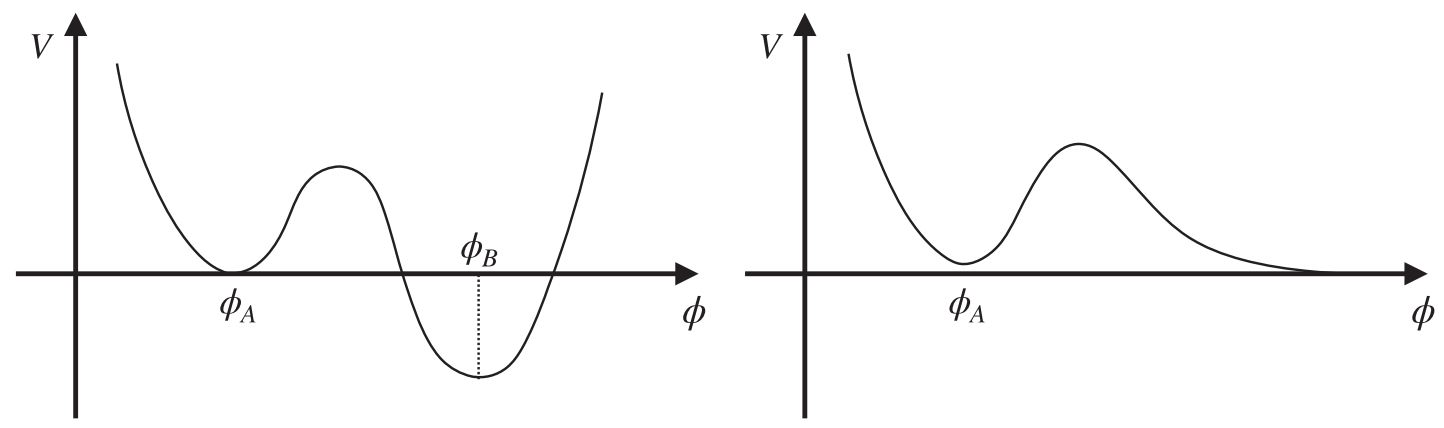

FIG. 1. Potential. 
next section, we can work in a compact space such as a three-sphere.

At this point we can write, in general

$$
\begin{array}{ll}
k=\sqrt{2[E-U[\phi(\tau)]]}, & \text { for } E>U(\phi(\tau)), \\
\kappa=\sqrt{2[U[\phi(\tau)]-E]}, & \text { for } E<U(\phi(\tau)) .
\end{array}
$$

The leading order wave functionals in the classically nonallowed and allowed regions respectively are determined by the WKB matching conditions. Consider the case in which the classically forbidden region is located at $\tau>\tau_{0}$, then the wave functionals in the classically allowed and in the classically forbidden regions are respectively

$$
\begin{aligned}
\Psi[\phi]= & \frac{2 A}{\sqrt{k}} \cos \left(\int_{\tau}^{\tau_{0}} k(\phi(\tau)) d \tau-\frac{\pi}{4}\right) \\
& -\frac{B}{\sqrt{k}} \sin \left(\int_{\tau}^{\tau_{0}} k(\phi(\tau)) d \tau-\frac{\pi}{4}\right), \\
\Psi[\phi]= & \frac{A}{\sqrt{\kappa}} \exp \left(-\int_{\tau_{0}}^{\tau} \kappa(\phi(\tau)) d \tau\right) \\
& +\frac{B}{\sqrt{\kappa}} \exp \left(\int_{\tau_{0}}^{\tau} \kappa(\phi(\tau)) d \tau\right),
\end{aligned}
$$

If the classically forbidden region is located at $\tau<\tau_{0}$, the wave functionals are

$$
\begin{aligned}
\Psi[\phi]= & \frac{A}{\sqrt{\kappa}} \exp \left(-\int_{\tau_{0}}^{\tau} \kappa(\phi(\tau)) d \tau\right) \\
& +\frac{B}{\sqrt{\kappa}} \exp \left(\int_{\tau_{0}}^{\tau} \kappa(\phi(\tau)) d \tau\right), \\
\Psi[\phi]= & \frac{2 A}{\sqrt{k}} \cos \left(\int_{\tau}^{\tau_{0}} k(\phi(\tau)) d \tau-\frac{\pi}{4}\right) \\
& -\frac{B}{\sqrt{k}} \sin \left(\int_{\tau}^{\tau_{0}} k(\phi(\tau)) d \tau-\frac{\pi}{4}\right) .
\end{aligned}
$$

In the above we have only kept the prefactor corresponding to longitudinal fluctuations of the field i.e., corresponding to the first term in Eq. (2.27). The second term coming from transverse fluctuations is not explicitly written since it plays no role in the further discussion.

Now recall that the classically allowed and forbidden regions are defined in terms of the potential $U[\phi]$, that depends on the specific path in the field space chosen to perform the integration. Hence they cannot be visualized in the potentials of Fig. 1. However, we can expect that for a generic path in field space, it would take a form that is similar to that shown in Fig. 1, with a finite barrier in the middle and infinite barriers on both sides for the case of the left panel of Fig. 1, and on the left side only for the case of the right panel of Fig. 1, (see Fig. 2). At this point we will distinguish between the two cases.

\section{A. WKB for decay in a two-vacua potential}

In the case of the left panel of Fig. 2, we can identify five different regions, three of which are classically disallowed $(1,3$, and 5$)$ while two are classically allowed (2 and 4$)$. We impose that in region 1 the decaying component of the wave functional is absent, hence $B_{1}=0$ and

$$
\Psi_{1}[\phi]=\frac{A_{1}}{\sqrt{\kappa}} \exp \left(-\int_{\tau}^{a} \kappa d \tau\right)
$$

Using Eq. (3.8) we can easily find that

$$
\begin{aligned}
& A_{2}=\left(\cos \theta e^{-i \pi / 4}+\sin \theta e^{i \pi / 4}\right) A_{1}, \\
& B_{2}=\left(\cos \theta e^{i \pi / 4}+\sin \theta e^{-i \pi / 4}\right) A_{1},
\end{aligned}
$$

where $\theta=\int_{a}^{b} k d \tau$, so that

$$
\begin{aligned}
\Psi_{2}[\phi]= & \frac{A_{2}}{\sqrt{k}} \exp \left(i \int_{b}^{\tau} k d \tau\right) \\
& +\frac{B_{2}}{\sqrt{k}} \exp \left(-i \int_{b}^{\tau} k d \tau\right) .
\end{aligned}
$$

The connection between regions 2 and 4 can be easily found by using the following connection formula, (see [40])

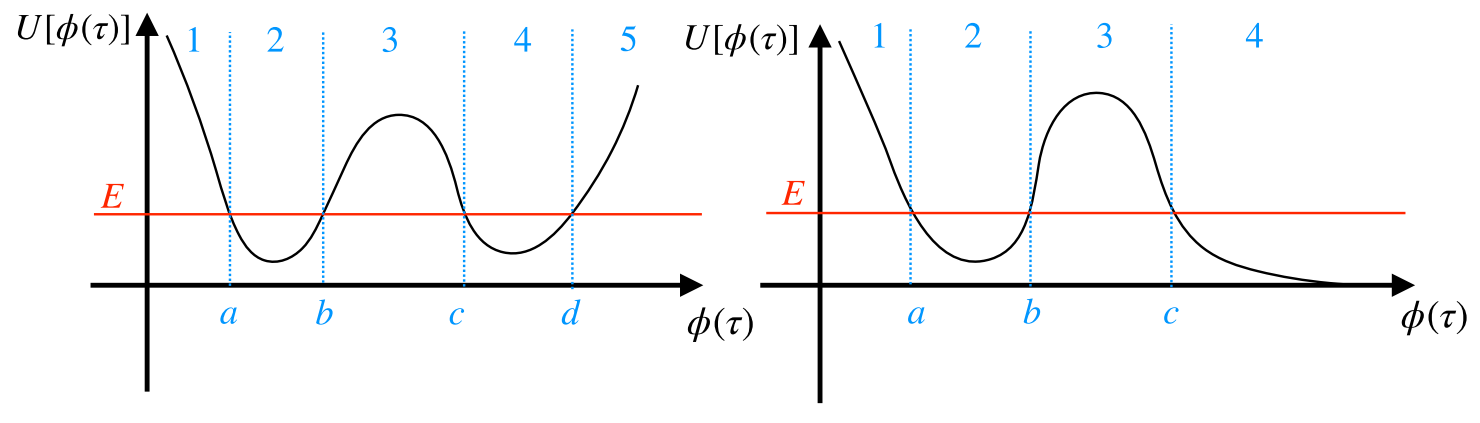

FIG. 2. Potential. 


$$
\left(\begin{array}{l}
A_{4} \\
B_{4}
\end{array}\right)=\frac{1}{2}\left(\begin{array}{cc}
\frac{1}{2 \lambda}+2 \lambda & i\left(\frac{1}{2 \lambda}-2 \lambda\right) \\
-i\left(\frac{1}{2 \lambda}-2 \lambda\right) & \frac{1}{2 \lambda}+2 \lambda
\end{array}\right)\left(\begin{array}{l}
A_{2} \\
B_{2}
\end{array}\right),
$$

where $\lambda=\exp \left(\int_{b}^{c} \kappa d \tau\right)$. We find that

$$
\begin{aligned}
& A_{4}=e^{i \pi / 4} \frac{A_{2}}{2 \lambda}\left(\sin \theta-4 i \cos \theta \lambda^{2}\right), \\
& B_{4}=e^{i \pi / 4} \frac{A_{2}}{2 \lambda}\left(-i \sin \theta+4 \cos \theta \lambda^{2}\right),
\end{aligned}
$$

so that the wave functional takes the form

$$
\begin{aligned}
\Psi_{4}[\phi]= & \frac{A_{4}}{\sqrt{k}} \exp \left(i \int_{c}^{\tau} k d \tau\right) \\
& +\frac{B_{4}}{\sqrt{k}} \exp \left(-i \int_{c}^{\tau} k d \tau\right) .
\end{aligned}
$$

Using Eq. (3.7) we find that

$$
A_{5}=\frac{A_{4} e^{i \omega}+B_{4} e^{-i \omega}}{2}, \quad B_{5}=i\left(A_{4} e^{i \omega}-B_{4} e^{-i \omega}\right),
$$

where $\omega=\int_{c}^{d} k d \tau$, so that

$$
\Psi_{5}[\phi]=\frac{A_{5}}{\sqrt{\kappa}} \exp \left(-\int_{d}^{\tau} \kappa d \tau\right)+\frac{B_{5}}{\sqrt{\kappa}} \exp \left(\int_{d}^{\tau} \kappa d \tau\right) .
$$

Of course, we need to require that the rising component of the wave functional is absent in region 5 , namely that $B_{5}=0$. This implies

$$
-4 \lambda^{2}\left(1+i e^{2 i \omega}\right) \cos \theta+i\left(1-i e^{2 i \omega}\right) \sin \theta=0,
$$

which is satisfied if

$$
\begin{array}{llll}
\cos \theta=0 & \text { and } \quad \omega=-\frac{\pi}{4}+n \pi \quad(n \in \mathbb{Z}), \quad \text { or } \\
\sin \theta=0 \quad \text { and } \quad \omega=\frac{\pi}{4}+n \pi \quad(n \in \mathbb{Z}) . &
\end{array}
$$

However, note that if we require that $\sin \theta=0$, the coefficients $A_{4}$ and $B_{4}$ in Eq. (3.13) would be enhanced with respect to $A_{2}$ and $B_{2}$ by a factor $\propto \lambda$. If the initial condition of the process is a homogeneous configuration with the field in the false vacuum, we expect that the coefficients $A_{4}$ and $B_{4}$ are suppressed with respect to $A_{2}$ and $B_{2}$, therefore we need to impose $\cos \theta=0$. Note that the leading order of the transmission coefficient $T^{2}=\frac{\left|\Psi_{4}[\phi]\right|^{2}}{\left|\Psi_{2}[\phi]\right|^{2}}$ is given by the factor $1 / \lambda^{2}$ and gives a measure of the decay rate, despite in this case it is not possible to formally define it in terms of the $S$-matrix (unlike the case discussed in the next section). At leading order then

$$
\Gamma \sim T^{2} \sim \frac{1}{\lambda^{2}}=\exp \left(-2 \int_{b}^{c} \kappa d \tau\right)
$$

which is equivalent to the CDL result.

It is particularly interesting to notice that the result in Eq. (3.15) is equivalent to the Euclidean action evaluated on the bounce solution, upon subtraction of the background action. Let us make this statement more explicit: between the two turning points $b$ and $c$, the potential energy is larger than the total energy $E$ of the system. As the total energy is given by the sum of kinetic energy and potential energy, the kinetic energy has to be negative. This can be achieved by rotating the time variable to the Euclidean time, that gains an imaginary unit and makes the kinetic energy negative. Hence, in the under-the-barrier region (between $b$ and $c$ ) it is totally justified to rotate to Euclidean time $s=i t$, where $t$ is the usual Lorentzian time. The Euclidean action is

$$
\begin{aligned}
& S_{E}[\phi(s)] \\
& \quad=\int d s\left[\int d^{3} x\left(\frac{1}{2}\left(\frac{d \phi(s)}{d s}\right)^{2}\right)+U[\phi(s)]\right] .
\end{aligned}
$$

The Euclidean energy is conserved, which implies

$$
\int d^{3} x\left(\frac{1}{2}\left(\frac{d \phi(s)}{d s}\right)^{2}\right)-U[\phi(s)]=-E,
$$

where we take $E=U\left[\phi_{A}\right]^{6}$ (despite the fact that the WKB argument holds for a general $E$ ), assuming that the initial state is a homogeneous field configuration $\phi=\phi_{A}$. Using Eq. (3.17), and noting that, in the under-the-barrier region

$$
\frac{d \tau}{d s}=\sqrt{2\left(U[\phi(s)]-U\left[\phi_{A}\right]\right)},
$$

the Euclidean action in Eq. (3.16) becomes simply

$$
\begin{aligned}
S_{E}[\phi(s)] & =\int d s\left[\int d^{3} x\left(\frac{1}{2}\left(\frac{d \phi(s)}{d s}\right)^{2}\right)+U[\phi(s)]\right] \\
& =\int d s\left[2\left(U[\phi(s)]-U\left[\phi_{A}\right]\right)\right]+\int d s U\left[\phi_{A}\right] \\
& =\int_{b}^{c} d \tau \sqrt{2\left(U[\phi(s)]-U\left(\phi_{A}\right)\right)}+S_{E}^{\text {back }},
\end{aligned}
$$

where $S_{E}^{\text {back }}$ is the background Euclidean action evaluated on the homogeneous solution $\phi=\phi_{A}$. Therefore

$$
S_{E}[\phi(s)]-S_{E}^{\mathrm{back}}=\int_{b}^{c} d \tau \kappa
$$

which shows the equivalence between the Euclidean action evaluated on the bounce solution (upon subtracting

\footnotetext{
${ }^{6}$ Note that in the case of the left panel of Fig. 1, $E=U\left[\phi_{A}\right]=0$.
} 
the background action) and the usual WKB factor of Eq. (3.15).

Of course, the resort to the use of the Euclidean action is just a trick that sometimes makes the computation easier and is completely justified quantum mechanically, as it reproduces exactly the WKB result. However, there is no intrinsic reason to use the bounce solution for the postnucleation phase; up to Eq. (3.15) we have not introduced Euclidean time and the Euclidean action, and these are actually not needed to get to the final result. The quantum mechanics problem only knows about the symmetries that are put in the problem from the very beginning; for instance one can require from the start that the problem has a spherical $O(3)$ symmetry. There is no way for the postnucleation solution to gain a $\sqrt{t^{2}-|x|^{2}}$ dependence, as it is usually obtained by naively rotating back to Lorentzian signature the bounce solution.

\section{B. WKB for decay in run-away potential}

In the case of the right panel of Fig. 1 we can give an explicit definition of the decay rate, as we can discuss the question in terms of an $S$-matrix. We have in mind a state which comes in from the right, tunnels through the barrier, is reflected off the wall on the left and tunnels through the barrier back to give an outgoing state to the right. The resonances that can be formed in this process constitute the decaying state that we are interested in. The $S$-matrix is a phase $\left(S=A_{4} / B_{4}\right)$ with complex poles corresponding to the bound states in region 2 . To identify the decay widths of the corresponding resonances we first identify the bound states; these correspond to

$$
\cos \theta=0 \Rightarrow \theta=\left(n+\frac{1}{2}\right) \pi \quad(n \in \mathbb{Z}) .
$$

Note that the last expression determines the possible discrete values of the energy $E_{n}$; in fact the energy appears both in the limits of integration (as it determines the turning points) and in the integrand [see Eq. (3.5) and Eq. (3.6)]. Considering the lowest energy state $E_{0}$ and expanding around this point we can write (see [40])

$$
\left.\cos \theta \simeq \mp\left(E-E_{0}\right)\left(\frac{\partial \theta}{\partial E}\right)\right|_{E=E_{0}},\left.\quad \sin \theta\right|_{E=E_{0}} \simeq 1,
$$

and get

$$
\begin{aligned}
S & \equiv \frac{A_{4}}{B_{4}}=\frac{E-E_{0}-i\left[1 /\left(\left.4 \theta^{2}\left(\frac{\partial \theta}{\partial E}\right)\right|_{E=E_{0}}\right)\right]}{E-E_{0}+i\left[1 /\left(\left(\left.4 \theta^{2}\left(\frac{\partial \theta}{\partial E}\right)\right|_{E=E_{0}}\right)\right]\right.} \\
& \equiv e^{2 i \phi}=\frac{E-E_{0}-i \Gamma / 2}{E-E_{0}+i \Gamma / 2} .
\end{aligned}
$$

with the decay width given by

$$
\Gamma=\left.\frac{1}{2 \lambda^{2}}\left(\frac{\partial \theta}{\partial E}\right)^{-1}\right|_{E=E_{0}},
$$

where as defined earlier $\lambda=\exp \left(\int_{b}^{c} \kappa d \tau\right)$. The phase shift $\phi$ has then the standard form

$$
\tan \phi=\frac{\Gamma / 2}{E-E_{0}},
$$

with the lifetime of the resonance given by

$$
\begin{aligned}
\Gamma^{-1}= & \left.2 \lambda^{2}\left(\frac{\partial \theta}{\partial E}\right)\right|_{E=E_{0}} \\
= & {\left[\left.\frac{\partial}{\partial E} \int_{a}^{b} d \tau \sqrt{2(E-U(\tau))}\right|_{0}\right] } \\
& \times 2 \exp \left[2 \int_{b}^{c} d \tau \sqrt{2(U(\tau)-E)}\right] .
\end{aligned}
$$

Note that $\left.2\left(\frac{\partial \theta}{\partial E}\right)\right|_{E=E_{0}}$ is the classical period for oscillations between $a$ to $b$ and one could have divided the transition probability i.e., the WKB factor in Eq. (3.15) to get this formula heuristically, while here we have derived it purely from quantum mechanical considerations.

\section{Comparison to Coleman's formula}

We showed above that the exponential term in the lifetime of the false vacuum (for the particular case where the energy corresponds to the energy at the minimum of the potential) is the same as in Coleman's calculation [see Eq.. (3.20)]. On the other hand the prefactor is quite different from that in the well-known formula derived by Coleman $[1,2]$.

(a) The tunneling rate $\Gamma$ given above is proportional to the frequency of oscillation of the field (with energy $E_{0}$ ) in the local minimum of the potential. In Coleman's picture the probability of tunneling has a infinite factor of time coming from the zero mode corresponding to time translations of the instanton which is then divided out to give a tunneling rate per unit time. Such a picture does not appear to come out of this direct WKB calculation.

(b) Similarly in Coleman's calculation there is an infinite (for noncompact space) volume factor which is also divided out to get a transition probability per unit volume. The above calculation has no such factorwhat we have instead is the analog of the decay rate of a resonance. For a compact space it gives the probability for the decay of the entire space-not the probability for bubble nucleation.

(c) The ground state energy $E_{0}$ of the resonance (false vacuum) is greater than the energy of the (local) minimum of the potential due to zero point fluctuations.

However the derivation in Coleman's papers is not quite a straightforward application of WKB quantum mechanics, 
but involves a series of additional assumptions whose status we discuss below.

\section{Some issues with Coleman's argument}

Let us summarize some of the problematic aspects of Coleman's original approach. ${ }^{7}$

(a) The Hamiltonian $H$ is Hermitian; its eigenvalues are necessarily real. Coleman makes essential use of the heuristic interpretation of $\Gamma$ as the imaginary part of an eigenvalue of $H$ and the dilute gas approximation in order to get the decay rate. The calculation leading to Eq. (3.24) used no such interpretation- $-\Gamma$ is extracted from the $S$-matrix for the scattering of a field configuration from a potential which can accommodate quasibound states (resonances).

(b) Coleman's calculation disagrees in the prefactor from the standard WKB calculation (i.e., essentially Eq. (3.24) with $U$ now being the quantum mechanical potential). In particular Coleman's formula for the decay rate $\Gamma$ involves dividing by the (infinite) translation symmetry of his instanton i.e., the prefactor for the transition probability contains a factor $T \rightarrow \infty$ that is divided out to get the rate. In the calculation above there is no such factor and effectively the division is by the classical period for oscillations in the potential well.

(c) The last factor of Eq. (3.24) is the square of the standard WKB decay amplitude and naturally is the same as in Coleman's formula with the identification of the exponent as the difference between the Euclidean classical action for a classical solution with the appropriate boundary conditions, and the action for a particle whose position is localized in the well (at its minimum).

(d) In the field theory case Coleman gives an argument for the dominant contribution to this last factor to come from a Euclidean four-sphere configuration. However in the actual evaluation of the decay amplitude the thin-wall approximation is used in which the under-the-barrier region effectively shrinks to a brane. On the other hand propagation in the classically allowed region to the right of the barrier is described in terms of the analytic continuation of this Euclidean instanton. The justification for the latter is unclear.

(e) Of course one would like to have a quantum field theory argument for a physical picture of a first order phase transition with bubble nucleation and percolation. Coleman's argument is clearly motivated by this and indeed it would be nice to have a rigorous QFT justification for this. Unfortunately we do not see at this point how to achieve this.

\footnotetext{
${ }^{7}$ See [6] for related critical comments.
}

\section{VACUUM DECAY IN CURVED SPACE}

\section{A. Review of Coleman-De Luccia}

The application of Coleman's arguments to the case involving gravity are even more problematic. Apart from the issues highlighted above there is the problem that the notion of time (and hence that of transition probability per unit time) needs to be reinterpreted given that the WDW equation does not admit the usual notion of time. Let us first discuss the Euclidean minisuperspace case as given by CDL for the case $d S \rightarrow \mathcal{M}$ and generalized to $d S \rightarrow d S$ by Parke [41]. Putting $t=i \tau$ and $a(t)=\rho(\tau)$ and gauge fixing to $N=1$ we have the metric $d s^{2}=d \tau^{2}+\rho^{2}(\tau) d \Omega_{3}^{2}$. The relevant Euclidean equation of motion then is the $\tau \tau$ component of the Einstein equation which reads $\left(\rho^{\prime}=d \rho / d \tau\right)$

$$
\rho^{2}=1+\frac{1}{3}\left(\frac{1}{2} \phi^{2}-V(\phi) \rho^{2}\right)
$$

The Euclidean action is

$$
\begin{aligned}
S_{E} & =-2 \pi^{2} \int_{0}^{\tau_{\max }} d \tau\left[3 \rho+3 \rho \rho^{2}-V \rho^{3}-\rho^{3} \frac{1}{2} \phi^{\prime 2}\right] \\
& =-12 \pi^{2} \int_{0}^{\tau_{\max }} d \tau\left[\rho-\frac{1}{3} V \rho^{3}\right] .
\end{aligned}
$$

In the last step we used the equation of motion in Eq. (4.1). In this Euclidean argument this is supposed to be the instanton (bounce) action with $\phi(0)=\phi_{B}$ i.e., the value of the field at the so-called true minimum and $\phi(\infty)=\phi_{A}$, the value of the field in the false minimum. Of course there is complete symmetry between the two so the bounce action is the same for going from $A \rightarrow B$ or $B \rightarrow A$. The difference between up-tunneling (true to false vacuum) and downtunneling (false to true) just comes from the fact that the background action which is subtracted to get the tunneling amplitude is different. So in the case of down-tunneling that we will consider here (i.e., $A \rightarrow B$ ) the tunneling amplitude is given by $e^{B / 2}$ where,

$$
\frac{B}{2}=S_{E}-S_{E}^{A}
$$

where the second term is the action for $\phi$ remaining at the false minimum $\phi_{A}$. i.e.,

$$
S_{E}^{A}=-12 \pi^{2} \int_{0}^{\tau_{\max }} d \tau\left[\rho-\frac{1}{3} V_{A} \rho^{3}\right],
$$

where $V_{A}=V\left(\phi_{A}\right)$. Hence we have 


$$
\begin{aligned}
\frac{B}{2} & =-12 \pi^{2} \int_{0}^{\tau_{\max }} d \tau\left[\rho-\frac{1}{3} V \rho^{3}\right]+12 \pi^{2} \int_{0}^{\tau_{\max }} d \tau\left[\rho-\frac{1}{3} V_{A} \rho^{3}\right] \\
& =-12 \pi^{2} \int_{0}^{\bar{\tau}-\delta \tau} d \tau\left[\rho-\frac{1}{3} V_{B} \rho^{3}\right]+2 \pi^{2} \bar{\rho}^{3} T \\
& +12 \pi^{2} \int_{0}^{\bar{\tau}-\delta \tau} d \tau\left[\rho-\frac{1}{3} V_{A} \rho^{3}\right]
\end{aligned}
$$

In the second line we have assumed that beyond the point $\bar{\tau}+\delta \tau, V \simeq V_{A}$ so that the contribution from $\bar{\tau}+\delta \tau$ to $\tau_{\max }$ in the first term of the first line cancels against the second term. Also $T$ in the middle term is defined by

$$
\bar{\rho}^{3} T=2 \int_{\bar{\tau}-\delta \tau}^{\bar{\tau}+\delta \tau} d \tau \rho^{3}\left(V\left(\phi(\tau)-V_{A}\right) .\right.
$$

In the second line of Eq. (4.4) we have taken the path in $\tau$ such that for $0<\tau \leq \bar{\tau}-\delta \tau, \phi$ is held fixed at $\phi_{B}$ while in the interval $\bar{\tau}+\delta \tau \leq \tau<\tau_{\max }, \phi=\phi_{A}$. So in the first and third terms in Eq. (4.4) we can replace the integral over $d \tau=\frac{d \tau}{d \rho} d \rho$ using the Euclidean Eq. (4.1) with $\phi$ fixed. ${ }^{8}$ This gives $\frac{d \tau}{d \rho}= \pm 1 / \sqrt{1-V_{B, A} \rho^{2}}$ in the first and third terms ${ }^{9}$ so these integrations can be done giving us (in the thin-wall limit $\delta \tau \rightarrow 0$ ),

$$
\begin{aligned}
\frac{B}{2}= & -12 \pi^{2}\left[ \pm \frac{\left(1-\frac{1}{3} V_{A} \bar{\rho}^{2}\right)^{3 / 2}-1}{V_{A}} \mp \frac{\left(1-\frac{1}{3} V_{B} \bar{\rho}^{2}\right)^{3 / 2}-1}{V_{B}}\right] \\
& +2 \pi^{2} \bar{\rho}^{3} T .
\end{aligned}
$$

$\bar{\rho}$ is then determined by extremizing $B$. Upon substituting this value into the above one then gets the usual expressions which we will quote later after rederiving the above without invoking Euclidean arguments with their corresponding interpretational issues.

\section{B. Vacuum transitions in minisuperspace}

An instructive exercise, that helps understanding the formalism outlined in Sec. II and shows the differences between the Lorentzian and Euclidean appproaches, consists in studying vacuum transitions in a minisuperspace setup that includes a real scalar field. This calculation is a generalization of the 'tunneling from nothing' scenario [20-23]. For a recent discussion see for instance [42-44]. The metric is

$$
d s^{2}=-N^{2}(t) d t^{2}+a^{2}(t)\left(d r^{2}+\sin ^{2} r d \Omega_{2}^{2}\right) .
$$

The action (setting $M_{p}=1 / \sqrt{8 \pi G}=1$ ) is given by the sum $S=S_{g}+S_{m}$, where

\footnotetext{
${ }^{8}$ Although not explicitly stated this seems to have been assumed also in [41].

${ }^{9}$ In [41] only the positive sign is kept here.
}

$$
\begin{array}{r}
S_{g}=2 \pi^{2} \int_{0}^{1} d t\left(-N^{-1} 3 a \dot{a}^{2}+3 k a N\right), \\
S_{m}=2 \pi^{2} \int_{0}^{1} d t\left(N^{-1} \frac{1}{2} a^{3} \dot{\phi}^{2}-N a^{3} V(\phi)\right) .
\end{array}
$$

Here $k= \pm 1,0$ depending on whether the three-spatial slice is positively (negatively) curved or flat. Of course in the open $k=0,-1$ cases the factor $2 \pi^{2}$ would have to be replaced by an appropriate compactified volume factor and the spatial metric in Eq. (4.7) would need to be replaced by a flat or hyperbolic metric. Here we will focus on the $k=$ +1 case and for convenience we will drop the $2 \pi^{2}$ factor in the calculations below and restore it in the expressions for the classical action. We will make some remarks at end on the other two cases. The canonical momenta are

$\pi_{N}=0, \quad \pi_{a}=-N^{-1} 6 a \dot{a}, \quad \pi_{\phi}=N^{-1} a^{3} \dot{\phi}$,

and the Hamiltonian constraint is

$$
\mathcal{H}=N\left(-\frac{\pi_{a}^{2}}{12 a}+\frac{\pi_{\phi}^{2}}{2 a^{3}}-3 a+a^{3} V(\phi)\right) \approx 0 .
$$

Comparing with Eq. (2.4) we have

$$
\begin{gathered}
G^{a a}=-\frac{1}{6 a}, \quad G^{\phi \phi}=\frac{1}{a^{3}}, \\
f(a, \phi)=-3 a+a^{3} V(\phi) .
\end{gathered}
$$

Consider a scalar potential with two dS minima in $\phi_{A}$ and $\phi_{B}$, with $V\left(\phi_{A}\right) \equiv V_{A}>V_{B} \equiv V\left(\phi_{B}\right)$. Then the general shape of the function $-f(a, \phi)$ in Eq. (4.13) is plotted in Fig. 3.

As we emphasized before, the superspace metric is not positive definite in the presence of gravity and this introduces significant differences to WKB type tunneling arguments. As can be seen from this constraint equation in the absence of the scalar field (this is the 'tunneling from

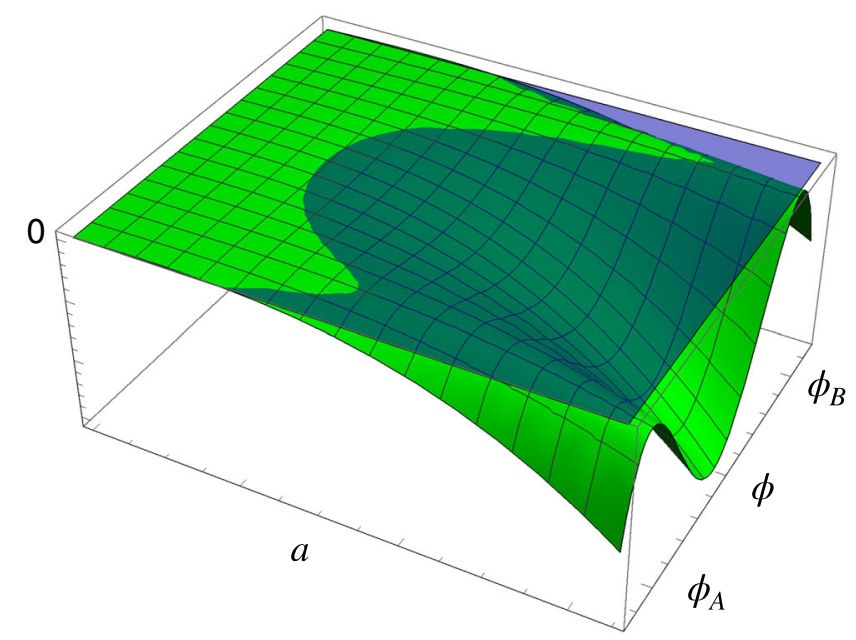

FIG. 3. The function $-f(a, \phi)$ for a potential with two $\mathrm{dS}$ minima $\phi_{A}$ and $\phi_{B}$ and where $V\left(\phi_{A}\right)>V\left(\phi_{B}\right)$. 
nothing' model of [20-23]), one has a barrier at fixed $\phi$ for the scale factor $a$ when $a<\sqrt{3 / V}$ whereas if the geometry is fixed (as in the original investigations of Coleman et al.) then there is a barrier for $\phi$ when $V$ is greater than its value at the point where $\pi_{\phi}$ becomes zero. However it is clear that these simple situations are not the only possibilities when both $a$ and $\phi$ are present. The following is to our knowledge the first time this more complex situation is discussed.

\section{Recovering Coleman-De Luccia}

It is instructive to try and recover the CDL expression in Eq. (4.6) using the formalism of Sec. II. Hopefully, this might help us in better understanding CDL. Let us choose the deformation parameter $s$ (in analogy with the Euclidean time $\tau$ ) such that from some initial value (say at $s=0$ ) to the point $\bar{s}-\delta s$ the field $\phi$ remains very close to $\phi_{B}$ and for points $s_{\max }>s>\bar{s}+\epsilon, \phi$ becomes close to $\phi_{A}$. It should be emphasized here that $s$ has nothing to do with real time-it is simply a deformation parameter that parametrizes the path of integration. The range $\bar{s}-\epsilon<s<\bar{s}+\epsilon$ is the transitional region where in effect CDL used the thin-wall approximation. Thus the classical action [see Eq. (2.11)] is

$$
\begin{aligned}
S_{0}\left(a_{0}, \phi_{B} ; a_{\max }, \phi_{A}\right) \\
=-12 \pi^{2} \int_{0}^{s_{\max }} d s^{\prime} C^{-1}\left(s^{\prime}\right)\left(-a+a^{3} \frac{V(\phi)}{3}\right) \\
=-12 \pi^{2} \int_{0}^{\bar{s}-\delta s} d s^{\prime} C^{-1}\left(s^{\prime}\right)\left(-a+a^{3} \frac{V_{B}}{3}\right) \\
\quad-12 \pi^{2} \int_{\bar{s}-\delta s}^{\bar{s}+\delta s} d s^{\prime} C^{-1}\left(s^{\prime}\right)\left(-a+a^{3} \frac{V(\phi)}{3}\right) \\
\quad-12 \pi^{2} \int_{s+\delta s}^{s_{\max }} d s^{\prime} C^{-1}\left(s^{\prime}\right)\left(-a+a^{3} \frac{V_{A}}{3}\right) .
\end{aligned}
$$

Here in the first term we have used the fact that $\phi$ remains constant and equal to $\phi_{B}$ while in the last term it remains equal to $\phi_{A}$.

This path corresponds to the Euclidean path chosen by CDL and Parke. Thus in the Lorentzian case this action corresponds to 'tunneling from nothing', as in HartleHawking/Vilenkin-Linde [20,21,23] wave function of the universe arguments, essentially keeping $\phi$ fixed, to the potentially emergent state $B$ (the true vacuum in CDLs language), then making a transition to the state $A$ (where both $\phi$ and $a$ can change), that then emerges as the classical background space time. This is then to be compared to the situation where the state $A$ emerges from a 'tunneling from nothing' process. The latter gives an action

$$
\begin{aligned}
& S_{0}\left(a_{\max }, \phi_{A} ; a_{0}, \phi_{A}\right) \\
& \quad=-12 \pi^{2} \int_{0}^{s} d s^{\prime} C^{-1}\left(s^{\prime}\right)\left(-a+a^{3} \frac{V_{A}}{3}\right) .
\end{aligned}
$$

Now we have from Eq. (2.15)

$$
\begin{aligned}
\left(\frac{d \tau}{d s}\right)^{2} & =-6 a\left(\frac{d a}{d s}\right)^{2}+2 a^{3}\left(\frac{d \phi}{d s}\right)^{2} \\
& =-2 C^{-2}(s)\left(-3 a+a^{3} V(\phi)\right) .
\end{aligned}
$$

Thus as long as $a^{2}<3 / V(\phi)$, for a "timelike" trajectory in field space $C^{2}=-1$. In particular this would be the case for $d \phi / d s=0$. For the moment though we will leave this undetermined.

The transition probability is given by (ignoring the prefactors for the moment),

$$
\begin{aligned}
P(A \rightarrow B) & =\left|\frac{\Psi\left(a_{0}, \phi_{B} ; a_{\max }, \phi_{A}\right)}{\Psi\left(a_{0}, \phi_{A} ; a_{\max }, \phi_{A}\right)}\right|^{2} \\
& =\left|\frac{\alpha e^{i S_{0}\left(a_{0}, \phi_{B} ; a_{\max }, \phi_{A}\right)}+\beta e^{-i S_{0}\left(a_{0}, \phi_{B} ; a_{\max }, \phi_{A}\right)}}{\alpha e^{i S_{0}\left(a_{0}, \phi_{A} ; a_{\max }, \phi_{A}\right)}+\beta e^{-i S_{0}\left(a_{0}, \phi_{A} ; a_{\max }, \phi_{A}\right)}}\right|^{2} \\
& \equiv e^{-B} .
\end{aligned}
$$

The dominant term in this ratio will be exponentially larger than the subdominant terms so the latter may be safely ignored.

$$
\begin{aligned}
\frac{B}{2}= & i S_{0}\left(a_{0}, \phi_{B} ; a_{\max }, \phi_{A}\right)-i S_{0}\left(a_{0}, \phi_{A} ; a_{\max }, \phi_{A}\right) \\
= & -12 \pi^{2} i \int_{0}^{\bar{s}-\delta s} d s^{\prime} C^{-1}\left(s^{\prime}\right)\left(-a+a^{3} \frac{V_{B}}{3}\right) \\
& -12 \pi^{2} i \int_{\bar{s}-\delta s}^{\bar{s}+\delta s} d s^{\prime} C^{-1}\left(s^{\prime}\right)\left(-a+a^{3} \frac{V(\phi)}{3}\right) \\
& -12 \pi^{2} i \int_{\bar{s}+\delta s}^{s_{\max }} d s^{\prime} C^{-1}\left(s^{\prime}\right)\left(-a+a^{3} \frac{V_{A}}{3}\right) \\
& +12 \pi^{2} i \int_{0}^{s_{\max }} d s^{\prime} C^{-1}\left(s^{\prime}\right)\left(-a+a^{3} \frac{V_{A}}{3}\right),
\end{aligned}
$$

where we have chosen to keep $C$ so the choice of phase is so far undetermined. After some cancellations this may be rewritten as

$$
\begin{aligned}
\pm \frac{B}{2}= & -12 \pi^{2} i \int_{0}^{\bar{s}-\delta s} d s^{\prime} C^{-1}\left(s^{\prime}\right)\left(-a+a^{3} \frac{V_{B}}{3}\right) \\
& +12 \pi^{2} i \int_{0}^{\bar{s}-\delta s} d s^{\prime} C^{-1}\left(s^{\prime}\right)\left(-a+a^{3} \frac{V_{A}}{3}\right) \\
& +2 \pi^{2} \bar{a}^{3} T,
\end{aligned}
$$

where we have defined the tension $T$ in analogy with Eq. (4.5), as the contribution to the action coming from the portion of the path such that $d \phi / d s \neq 0$

$$
2 \pi^{2} \bar{a}^{3} T=12 \pi^{2} i \int_{\bar{s}-\delta s}^{s+\delta s} d s^{\prime} C^{-1}\left(s^{\prime}\right)\left(a^{3} \frac{V(\phi)-V_{A}}{3}\right) .
$$


Note that, despite the contribution in Eq. (4.20) is similar to Eq. (4.5), there is no physical wall in the process that we are considering, that preserves the full $O(4)$ symmetry of the minisuperspace model. So far we have not made any approximation. The terms in the first line of Eq. (4.19) will now be evaluated (as in the corresponding Euclidean case) keeping $\phi$ constant. So, we may use $\frac{d a}{d s^{\prime}}= \pm \sqrt{1-\frac{V_{A, B}}{3} a^{2}}$ [see Eq. (4.16) with $d \phi / d s=0$ which implies $C^{2}=-1$ ]. We will also assume that the last term of Eq. (4.19) is also integrated over a timelike path in field space so that we can choose $C^{2}=-1$ along this path as well, which requires of course that $d a / d s$ is nonzero along this path. Hence

$$
\begin{aligned}
S_{0}^{A, B} & = \pm i 12 \pi^{2} \int_{0}^{a} d a a \sqrt{\left(1-a^{2} \frac{V_{A, B}}{3}\right)} \\
& =\mp i 12 \pi^{2} \frac{1}{V_{A}}\left\{\left(1-a^{2} \frac{V_{A, B}}{3}\right)^{3 / 2}-1\right\}
\end{aligned}
$$

Thus we have [putting $a(\bar{s} \pm \delta s)=\bar{a} \pm \delta a$ ]

$$
\begin{aligned}
\pm \frac{B}{2}= & 12 \pi^{2}\left\{\frac{1}{V_{B}}\left[\left(1-(\bar{a}-\delta a)^{2} \frac{V_{B}}{3}\right)^{3 / 2}-1\right]\right. \\
& \left.-\frac{1}{V_{A}}\left[\left(1-(\bar{a}-\delta a)^{2} \frac{V_{A}}{3}\right)^{3 / 2}-1\right]\right\} \\
& +2 \pi^{2} \bar{a}^{3} T .
\end{aligned}
$$

\section{Concrete cases}

Let us now consider several cases to identify the transition amplitudes using our formalism.

(a) Thin-wall CDL Let us try to recover the CDL result in the thin-wall approximation. We can evaluate the last equation in the thin-wall approximation. In this case the potential essentially reduces to a brane so that effectively we may write the argument of the integral in Eq. (4.20) in the limit $\delta s \rightarrow 0$ as a delta function. Then the logarithm of the transition amplitude becomes

$$
\begin{aligned}
\pm \frac{B}{2}= & 12 \pi^{2}\left\{ \pm \frac{1}{V_{B}}\left[\left(1-\bar{a}^{2} \frac{V_{B}}{3}\right)^{3 / 2}-1\right]\right. \\
& \left.\mp \frac{1}{V_{A}}\left[\left(1-\bar{a}^{2} \frac{V_{A}}{3}\right)^{3 / 2}-1\right]\right\} \\
& +2 \pi^{2} \bar{a}^{3} T .
\end{aligned}
$$

This is the same expression as Eq. (4.6). It is also clear that this is the sum of two Hartle-Hawking/VilenkinLinde terms and ${ }^{10}$ and a term coming from the portion

\footnotetext{
${ }^{10}$ Note that in the thin-wall approximation the integrals in Eq. (4.5) and Eq. (4.20) imply that in the region around $\bar{s}$ the potential takes the form $a^{3}(s)\left(V(\phi(s))-V_{A}\right)=2 \bar{a}^{3} T \delta(s-\bar{s})$.
}

of the path where $d \phi / d s \neq 0$, that in the CDL computation corresponds to the wall tension contribution. However, notice that we recovered the CDL expression for the tunneling probability, Eq. (4.23) without having to go to Euclidean space, but just using WKB quantum mechanics and assuming that, for the path that extremizes the action, $C^{-1}$ in Eq. (4.20) is imaginary. Extremizing Eq. (4.23) gives the standard (generalized) CDL expression for transitions between two dS spaces (or AdS with appropriate sign changes). The extremum is at

$$
\begin{aligned}
\frac{1}{\bar{a}^{2}} & =\frac{V_{B}}{3}+\frac{1}{4}\left(\frac{2}{T} \frac{\Delta V}{3}+\frac{T}{2}\right)^{2} \\
& =\frac{V_{A}}{3}+\frac{1}{4}\left(\frac{2}{T} \frac{\Delta V}{3}-\frac{T}{2}\right)^{2},
\end{aligned}
$$

where $\Delta V=V_{A}-V_{B}$. Note that this expression shows that $\bar{a}$ is less than the horizon radius of both $A$ and $B$. Putting this into Eq. (4.23) gives the final expression (with $H^{2} \equiv V / 3$ )

$$
\begin{aligned}
B= & \pm 8 \pi^{2}\left[\frac{\left\{\left(H_{A}^{2}-H_{B}^{2}\right)^{2}+T^{2}\left(H_{A}^{2}+H_{B}^{2}\right)\right\} \bar{a}}{4 T H_{A}^{2} H_{B}^{2}}\right. \\
& \left.-\frac{1}{2}\left(H_{B}^{-2}-H_{A}^{-2}\right)\right] .
\end{aligned}
$$

This is of course the well-known result. However its derivation and interpretation is quite different from that of CDL (and subsequent work which generalized the CDL result). Firstly we did not explicitly use Coleman's tunneling formula-instead we directly solved the WDW equation in the classical approximation, as a deformation of the solution where the initial configuration is one in which the fields correspond to a dS space (with vacuum energy $V_{A}$ and compared it to the undeformed configuration). However, there are a few puzzles posed by this calculation as we discuss next.

(b) Hartle-Hawking interpretation As the last term in Eq. (4.23) is positive, it increases the absolute value of $B$ and hence decreases the tunneling probabilty. On the other hand, one can simply ask what is the relative probability of 'tunneling from nothing' to the state $B$ compared to 'tunneling from nothing' to the state $A$. In other words one might compute the following ratio

$$
\frac{B}{2}=\frac{\left|\Psi\left(a_{0}, \phi_{B} ; a_{\max }, \phi_{B}\right)\right|^{2}}{\left|\Psi\left(a_{0}, \phi_{A} ; a_{\max }, \phi_{A}\right)\right|^{2}},
$$

with $a_{\max } \geq \max \left(\sqrt{\left(3 / V_{A}\right)}, \sqrt{3 / V_{B}}\right)$. The result would then be given by the top line of Eq. (4.23) (i.e., the two Hartle-Hawking-Vilenkin-Linde terms) with no tension term. Note that, even though the 
integration of Eq. (4.23) in this case extends till $a_{\max }$, only the integrals up to $\sqrt{3 / V_{A}}$ and $\sqrt{3 / V_{B}}$ contribute to the real part of $B$. Hence, for instance, the factor $\left(1-\frac{\bar{a}^{2} V_{B}}{3}\right)^{3 / 2}$ evaluated at $\bar{a}^{2}>3 / V_{B}$ gives no contribution to $B$ and analogously for the term containing $V_{A}$ in Eq. (4.23). Hence the relative probability is now given by $P=e^{-B}$ with

$$
B=24 \pi^{2}\left\{\mp \frac{1}{V_{B}} \pm \frac{1}{V_{A}}\right\} .
$$

This is simply the ratio of the Hartle-HawkingVilenkin-Linde (depending on the choice of sign) probabilities for tunneling from 'nothing' to the state $B$ compared to tunneling from 'nothing' to the state $A$. Note that Eq. (5.11) might be interpreted as the tunneling rate for the transition $\mathrm{dS} A \rightarrow$ 'nothing' $\rightarrow$ $\mathrm{dS} B$ (or the opposite, depending on the choice of the signs). Interestingly, it seems that this would give a greater probability for transition than the CDL calculation. In this connection it should be pointed out that in the Hartle-Hawking case the wave function in the classical region is indeed a superposition of wave functions for expanding and contracting universes. The process we are envisaging may then be thought of as the contracting branch with the field sitting at the $A$ minimum tunneling to 'nothing' and then re-emerging as an expanding branch in the $B$ minimum or the reverse.

(c) CDL vs Hartle-Hawking In the Lorentzian analog of the CDL calculation on the other hand, it is not clear how the true vacuum (i.e., $B$ ) emerges into the classical region. In CDL, the tunneling rate is computed exactly with the same integral as in Eq. (4.18). However, in $\mathrm{CDL}$ this is interpreted as the action for a Euclidean configuration given by a compound state that joins two portions of four-spheres (corresponding to dS $B$ and $\mathrm{dS} A$ ) along a three-sphere (the wall); the $S O(5)$ symmetry of the Euclidean four dimensional dS is broken to $S O(4)$ by the presence of the 'Euclidean' wall. Afterwards, as described in the Appendix, one of the angular variables that implements the $S O(4)$ symmetry, is analytically continued and becomes the usual Lorentzian time, breaking the symmetry to $S O(1,3)$. In this way, the initial under-the-barrier Euclidean configuration (the compound state) becomes the real three-dimensional equal-time slice of the nucleated spacetime. As the continued angular variable is one that preserves the Euclidean $S O(4)$ symmetry, the nucleated spacetime is still a compound state of $\mathrm{dS} A$ and $\mathrm{dS} B$; both $\mathrm{dS}$ spacetimes enter the classical region and keep evolving according to the classical equations of motion. In our computation, the integral of Eq. (4.18) (that we chose in order to recover the same CDL expression, and try to give it a
Lorentzian interpretation) is associated with a particular path in field space that, starting from the configuration $\left(a_{0}, \phi_{B}\right)$ (' $B$ nothing') leads to the nucleation of a full dS $A$ sphere, i.e., the configuration $\left(a_{\max }, \phi_{A}\right)$. This has to be contrasted, as described in Eq. (4.17) with the path that, starting from the configuration $\left(a_{0}, \phi_{A}\right)$ leads to the same full dS $A$ sphere as above. Essentially we are comparing three-geometries, which is all one can do in the context of quantum gravity as described by the WDW equation. In our procedure we neither need a dilute gas approximation nor a single negative mode in the spectrum of fluctuations as in the CDL flat space argument. Furthermore, there is no notion of bubble nucleation. Both the numerator and the denominator in Eq. (4.17) correspond to spacetime configurations that preserve the $S O(4)$ symmetry. To be more explicit, the portion of the path that corresponds to the dS $B$ is always under the barrier in the Lorentzian case; the computation relies on the fact that $\bar{a}$ in Eq. (4.23) after extremizing is located in the under-the-barrier region, so that the integrands of Eq. (4.21) give an imaginary contribution and the resulting $B$ is real. In the Lorentzian approach, the $\mathrm{dS}$ spacetime $B$ never sees the light of the classical region. Hence, in the present case built on the CDL argument how $B$ emerges as a classical spacetime is not at all clear. Our computation relies on the fact that the path in field space extremising the action can be split as described in Sec. IV C, and that it is such that $C^{-1}$ in Eq. (4.20) is imaginary. If the latter condition holds, the portion of the path such that $d \phi / d s \neq 0$ contributes to the imaginary action, bringing a term which is analogous to Eq. (4.5) in the Euclidean computation. In general, this is not necessarily the case and one should compute the value of the action for the path that solves the equation of motion. In order to compute the contribution to the imaginary part of the action of a given path, it is sometimes more convenient to use the distance on field space as the parameter $s$, in which case the action for the wave function in the numerator of Eq. (4.17) can be written as

$$
\begin{aligned}
& S_{0}\left[a_{0}, \phi_{B} ; a_{\max }, \phi_{A}\right] \\
& =2 \pi^{2} \int_{a_{0}, \phi_{B}}^{a_{\max }, \phi_{A}}\left[-6 a d a^{2}+a^{3} d \phi^{2}\right]^{1 / 2} \\
& \quad \times\left[6 a\left(1-\frac{a^{2} V(\phi)}{3}\right)\right]^{1 / 2} .
\end{aligned}
$$

Hence, only the portions of the path such that the product of the two square roots in Eq. (4.28) is imaginary contribute to the imaginary part of the path, and hence to the tunneling rate. 

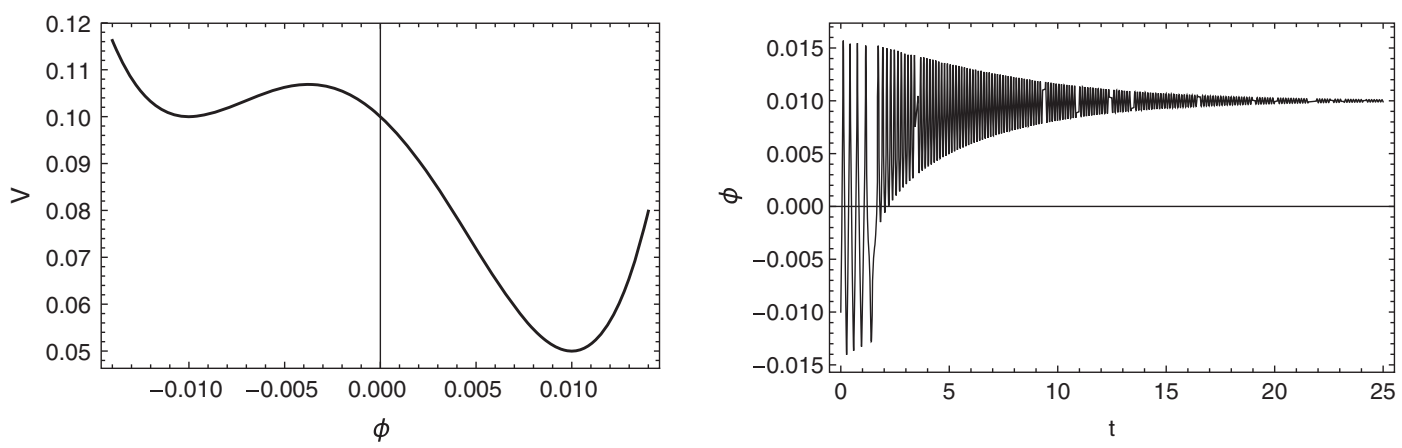

FIG. 4. The potential in the left panel is a quartic polynomial in which four parameters are fixed by requiring two minima as described in the main text, while the remaining parameter fixes the height of the barrier. The right panel shows the trajectory of the field that starts in the $A$ minimum and oscillates around the $B$ minimum with decreasing amplitude. Asymptotically it goes to rest in the $B$ minimum at $\phi=0.01$.

\section{E. Classical transitions in minisuperspace}

This subsection is somewhat orthogonal to the main thrust of this paper but we include it for completeness since it suggests additional possibilities for populating the landscape.

(a) Standard classical paths. We find it is interesting to notice that, due to the presence of a nonpositive definite superspace metric, it is possible to find a classical solution that connects two dS spacetimes $A$ and $B$. To illustrate this point, consider first the usual HartleHawking transition, as a special case of Eq. (4.28). If we include the scalar field, the model becomes richer. In fact, the first bracket in Eq. (4.28) is proportional to the kinetic terms of Eq. (4.11) and can become negative without resorting to an imaginary parameter

$$
\begin{aligned}
\frac{1}{2}\left(-6 a \dot{a}^{2}+a^{3} \dot{\phi}^{2}\right) & =-\frac{\pi_{a}^{2}}{12 a}+\frac{\pi_{\phi}^{2}}{2 a^{3}} \\
& =3 a\left(1-\frac{a^{2} V(\phi)}{3}\right) .
\end{aligned}
$$

Below, we show an example in which we observe a classical transition from $\mathrm{dS} A$ to $\mathrm{dS} B$ in the potential of the left panel of Fig. 4. The initial conditions are given by $a(0)=\sqrt{3 / V_{A}}, \phi(0)=\phi_{A}=-0.01, \dot{\phi}(0)=0.2$, so that the field goes over the barrier and start oscillating in the dS $B$ minimum at $\phi_{B}=0.01$, with decreasing amplitude. In the example, the maximum of the barrier is located at $\phi_{\max } \simeq-0.00375$ and the value of the potential is $V_{\max } \simeq 0.1068$. The initial speed necessary for the field to classically overcome the barrier and make the transition possible is roughly given by the height of the barrier $\Delta V=V_{\max }-V_{A} \simeq 0.0068$, so that $\frac{1}{2} \dot{\phi}^{2} \gtrsim \Delta V$. This is analogous to the recently proposed 'fly-over' scenario [45]. ${ }^{11}$ Asymptotically, the dS $B$

\footnotetext{
${ }^{11}$ We can equally get Hawking-Moss configurations after adjusting some of the parameters.
}

solution is recovered. In the example we used $V_{A}=0.1$ and $V_{B}=0.05$. In Fig. 5 we report the values of the brackets in Eq. (4.28), showing that they change sign at the same time even though the parameter $s$ of the evolution is always kept real.

(b) Non-standard classical paths. The Hamiltonian and momentum constraints can be expressed as the standard FLRW equation

$$
\left(\frac{\dot{a}}{a}\right)^{2}=\frac{8 \pi G}{3}\left(\frac{1}{2} \dot{\phi}^{2}+V(\phi)\right)-\frac{1}{a^{2}}
$$

and the scalar field equation

$$
\ddot{\phi}+3\left(\frac{\dot{a}}{a}\right) \dot{\phi}+V^{\prime}(\phi)=0
$$

We want to explore if there are paths in the $\phi, a$ space that can connect the two sides of the barrier for a scalar potential with more than one minimum. A simple path

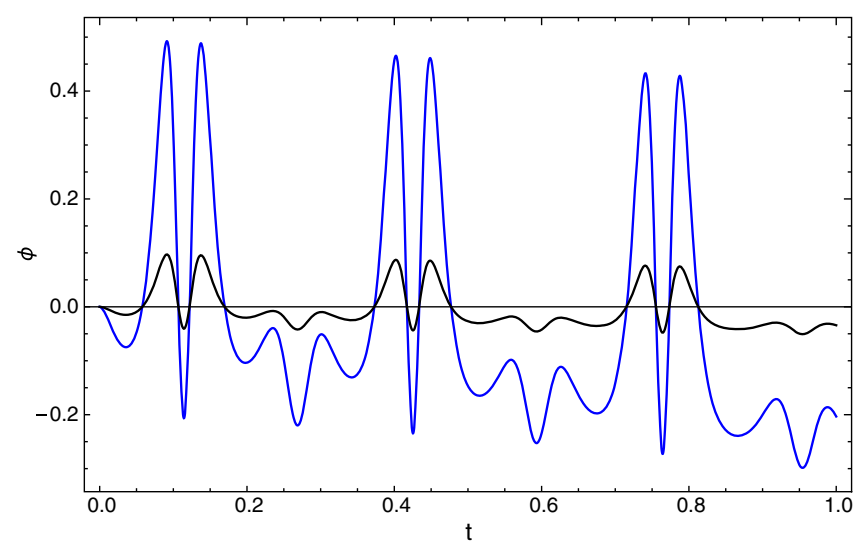

FIG. 5. The black line represents the term $\frac{1}{2}\left(a^{3} \dot{\phi}^{2}-6 a \dot{a}^{2}\right)$, while the blue line represents $1-\frac{a^{2} V(\phi)}{3}$. 

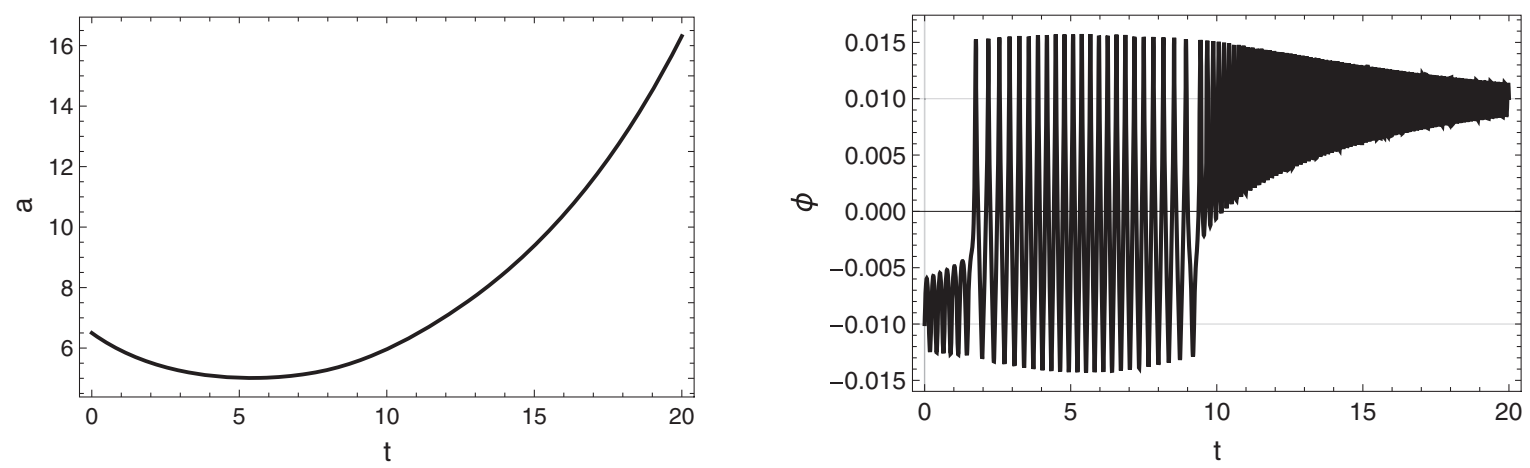

FIG. 6. In the left panel we plot the scale factor which is initially shrinking but then start growing. In the right panel we plot the value of the scalar field with respect to $t$. Initially the field starts oscillating around the higher vacuum, then it bounces between the two vacua and finally it settles in the lower vacuum.

can be starting with $a=$ constant. In this case the FLRW equation (Hamiltonian constraint) becomes

$$
\frac{1}{2} \dot{\phi}^{2}+V(\phi)=\frac{3}{8 \pi G a^{2}} \equiv E,
$$

which is the same as the energy conservation equation for a particle of energy $E$. As long as $E>V$ the field passes through classically over the barrier with no need of tunneling. Note that this happens only for a closed universe. Note also that the scalar field equation is satisfied automatically as usual for energy conservation. This is Einstein's static universe solution. For nonstatic cases, the standard classical solution would correspond to an initial velocity $\dot{\phi}$ to be big enough so that the kinetic energy can overcome the difference in potential energies to cross the barrier. In cosmological setting this would correspond to the standard scenarios such as Hawking-Moss and the fly-over of the previous bullet point. However considering trajectories in the $a(t), \phi(t)$ space can be more general than this. Due to the negative signature of the superspace metric there is no need to climb the potential energy barrier but we can move in the $2 \mathrm{~d}(a, \phi)$ space. For this, note that in Eq. (4.30) the curvature term adds a negative term to the scalar potential and therefore if the scale factor decreases with time the initial speed needed to cross the barrier is smaller than that required to compensate for the potential difference. This can also be seen from Eq. (4.31) since for a contracting universe $H<0$ the 'friction' term $3 H \dot{\phi}$ has the opposite sign, accelerating the field $\phi$ rather than slowing it down. This means that the classical path would have a contracting phase while the scalar field climbs through the barrier and then starts expanding after the transition. A sequence of contracting and expanding classical paths connecting different vacua would seem to be generic in a multi-minima scalar potential and could provide the basis of a novel scenario for early universe cosmology beyond old and new inflation. For a recent discussion of bouncing cosmologies see for instance [46].

\section{A numerical example}

Let us consider the scalar potential of Fig. 4 with different initial conditions. Taking $\phi(0)=\phi_{A}, a(0)=$ 6.5 and $\dot{\phi}(0)=0.1$, we find using the Friedmann equation that $\dot{a}(0) \simeq \pm 0.69$. Let us then consider a contracting universe as initial condition; $\dot{a}(0) \simeq-0.69$. In this case, we get the scale factor and field evolutions in Fig. 6 and Fig. 7. Note that this is a different scenario with respect to

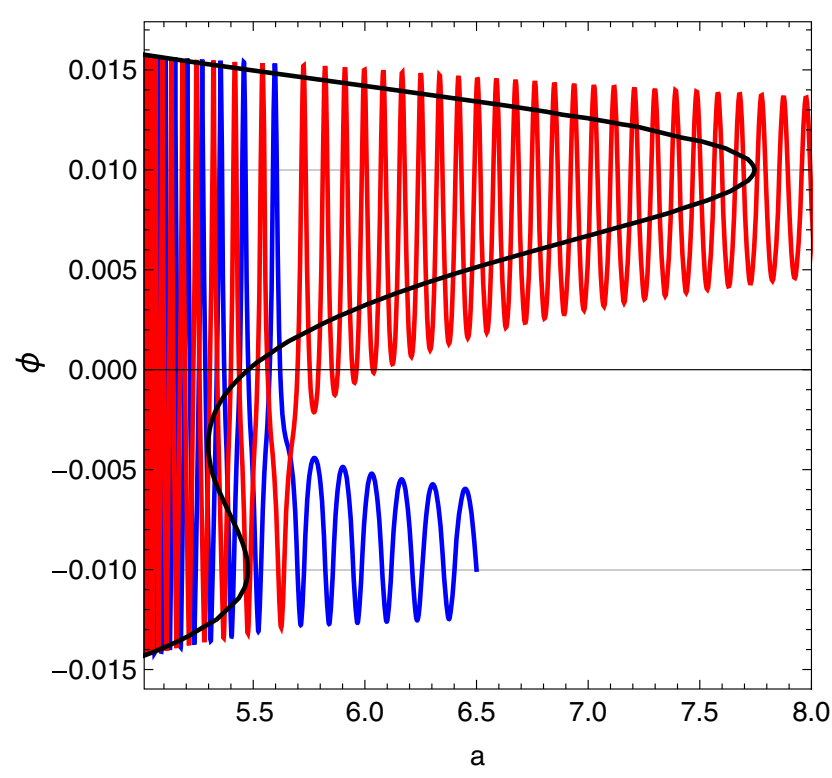

FIG. 7. We show the evolution of the system in field space: after a contracting phase (blue), the scale factor starts expanding (red) and the system gets trapped in the vacuum $B$. The black line corresponds to the locus $a^{2}=3 / V(\phi)$, hence it is the locus where the function $f(a, \phi)=0$, see Fig. 3 . 

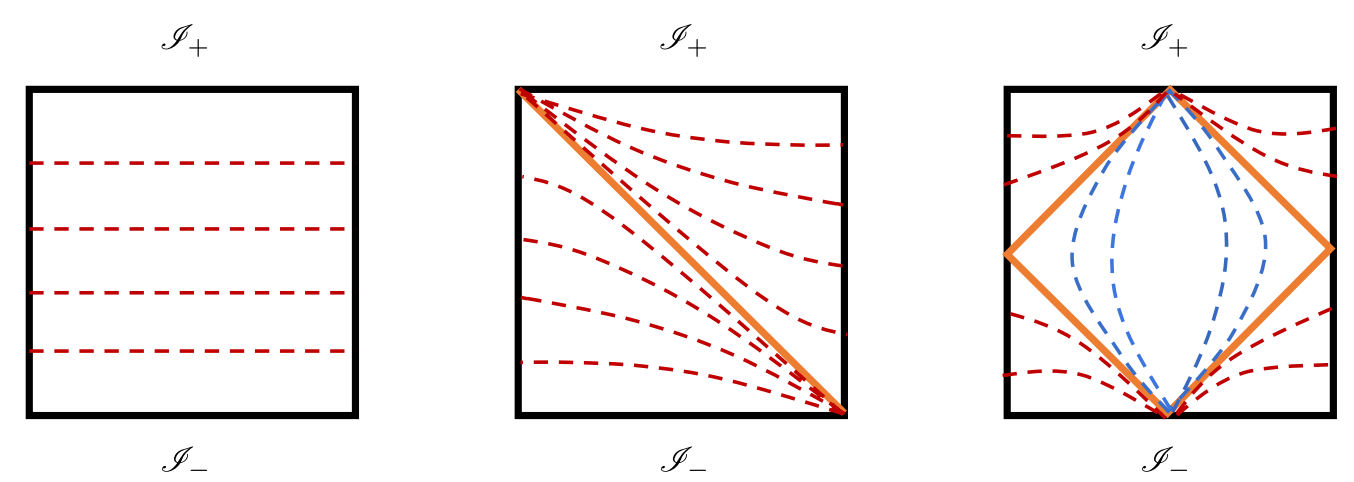

FIG. 8. Penrose diagrams for dS space with slicing corresponding from left to right to closed, flat and open slicings, respectively. Notice that the horizontal closed universe slicing is global.

standard paths through the barrier such as Hawking-Moss and 'fly-over' decay, as the initial kinetic energy $\left(\frac{1}{2} \dot{\phi}^{2} \simeq 5 \times 10^{-3}\right.$ in the present example) is smaller than the barrier height $\left(\Delta V \simeq 6.8 \times 10^{-3}\right)$. This illustrates explicitly our claim that due to the nonpositive nature of the superspace metric there are more configurations connecting the two different vacua. Of course to compare these classical paths to the quantum tansitions which are the main focus of this paper one should also discuss (as for example in [45]) what initial distribution of field velocities would be justified and we leave that to future work.

\section{OPEN OR CLOSED UNIVERSE?}

As we have seen in the previous sections, following a Lorentzian approach the spacetime geometry is established from the beginning. For simplicity and computational control we started with a closed universe and the end result is then also a closed universe with $k=1$ LFRW metric $^{12}$

$$
d s^{2}=-d t^{2}+a^{2}(t) d \Omega_{3}^{2},
$$

which differs clearly from the open universe conclusion of CDL. This metric is by construction Lorentzian and has the $O(4)$ symmetry corresponding to a closed universe with no need of any analytic continuation. For $a \propto \cosh (\lambda t)$ this corresponds to the $S O(4,1)$ invariant closed dS universe in global coordinates. In this case the natural foliation corresponds to horizontal surfaces of constant time.

Here it is important to remark that $\mathrm{dS}$ space allows open, flat and closed slicings, see Fig. 8. Therefore, geometrically all slicings are equally allowed. Which foliation is preferred

\footnotetext{
${ }^{12}$ Note that this is also the starting point in the Hartle-Hawking and Vilenkin approaches towards defining the wave function of the universe. The fact that closed universes are finite whereas flat and open universes have infinite volume makes closed universes better suited to define the probabilities associated to wave functions.
}

depends on the coupling to matter. For instance slices of constant inflaton field would naturally determine the proper time slicing and fix the curvature of the expanding universe within the nucleated bubble. In CDL this fixes the open universe but only after analytic continuation for which the original $O(4)$ symmetry becomes $O(3,1)$ of the open slicing. In the Lorentzian minisuperspace approach that we have followed here, the original $O(4)$ symmetry remains and implies the closed slicing. Next we will see that this remains true beyond minisuperspace as in the FMP Hamiltonian approach to quantum transitions.

\section{A. Beyond minisuperspace}

We have seen that our Lorentzian treatment led naturally to a closed slicing of dS space contrary to the CDL arguments. However, this may be an artefact of using the minisuperspace approximation in which the metric is only a function of time and there is no concrete description of the emergence of a bubble. In the Euclidean approach, even though the original calculations are also in minisuperspace, the presence of the bubble and its spacetime trajectory after tunneling is obtained from the proposed analytic continuation. In the Lorentzian approach there is at present no explicit formalism to describe the quantum transitions between different vacua of a scalar field potential. However, in the thin wall approximation in which the relevant quantities are the vacuum energy of the two vacua, the Hamiltonian formalism developed by Fischler, Morgan and Polchinski [8] can be used. In this case the spherically symmetric metric depends on both time and the radial coordinate. This allows us to describe the dynamics of the wall and its trajectory. Here we recall the basics of this approach, which like the Lorentzian minisuperspace model, naturally implies a closed universe slicing of the spacetime as seen by an observer inside the bubble.

The spherically symmetric metric takes the form:

$$
\begin{aligned}
d s^{2}= & -N_{t}^{2}(t, r) d t^{2}+L^{2}(t, r)\left(d r+N_{r}(t, r) d t\right)^{2} \\
& +R^{2}(t, r) d \Omega_{2}^{2},
\end{aligned}
$$


with $N_{t}, N_{r}$ the lapse and shift functions respectively and $d \Omega_{2}^{2}$ the line element for the two-sphere. The system consists of two dS spaces with cosmological constants $\Lambda_{I}, \Lambda_{O}$ separated by a wall of tension $\sigma$ at $r=\hat{r}$. The bulk and boundary actions are the standard gravitational ones and the matter action is given by the two cosmological constants, so the total action is

$$
\begin{aligned}
S= & \frac{1}{16 \pi G} \int_{\mathcal{M}} d^{4} x \sqrt{-g} \mathcal{R} \\
& +\frac{1}{8 \pi G} \int_{\partial \mathcal{M}} d^{3} y \sqrt{-h} K+S_{M}+S_{W},
\end{aligned}
$$

where $K$ is the extrinsic curvature of the wall and

$$
\begin{aligned}
& S_{M}=-4 \pi \int d t d r L N_{t} R^{2}\left(\Lambda_{O} \theta(r-\hat{r})+\Lambda_{I} \theta(\hat{r}-r)\right), \\
& S_{W}=-4 \pi T \int d t d r \delta(r-\hat{r})\left[N_{t}^{2}-L^{2}\left(N_{r}+\dot{\hat{r}}\right)^{2}\right] .
\end{aligned}
$$

In the above we defined $T \equiv 4 \pi G \sigma$. Following the standard Dirac prescription for this Hamiltonian system, the Hamiltonian and momentum constraints can be found and the matching conditions at the wall lead to an equation for the wall trajectory of the form

$$
\dot{\hat{R}}^{2}+V=-1 ; \quad V=-\frac{\hat{R}^{2}}{R_{0}^{2}},
$$

where $\hat{R}=R(\hat{r})$ and $R_{0}$ is the turning point

$$
R_{0}^{2}=\frac{4 T^{2}}{\left[\left(H_{O}^{2}-H_{I}^{2}\right)^{2}+2 T^{2}\left(H_{O}^{2}+H_{I}^{2}\right)+T^{4}\right]},
$$

with $H_{I, O}^{2}=8 \pi G \Lambda_{I, O} / 3$. The classical trajectory of the wall is then given by

$$
R(t)=R_{0} \cosh \frac{t}{R_{0}} .
$$

The quantum probabilities are determined from the solutions of the WDW equation $\mathcal{H} \Psi=0$ with $\mathcal{P}$ the relative probability of the configuration of the two dS spaces and the wall compared to that for just one $\mathrm{dS}$,

$$
\mathcal{P}(\mathrm{dS} \rightarrow \mathrm{dS} / \mathrm{dS} \oplus \mathrm{W})=\frac{|\Psi(\mathrm{dS} / \mathrm{dS} \oplus \mathrm{W})|^{2}}{|\Psi(\mathrm{dS})|^{2}} .
$$

The detailed calculation using the WKB method including a discussion of the matching of the under-the-barrier wave function to that in the classical region is given in [16] and the result reproduces the standard exponential factor $e^{-B}$ with $B$ given by Eq. (4.25). This provides yet another Lorentzian way to derive the same decay rate. But contrary to the minisuperspace approach, the presence of the wall and its classical trajectory after the transition is made quite explicit

$$
\Psi=a e^{I}+b e^{-I},
$$

where, given a configuration with action $S$, we have denoted the combination $i S=I$ and the action $S$ is evaluated on a classical solution. The total action away from the turning point (but still under the barrier) is

$$
\begin{aligned}
I_{\mathrm{tot}}= & \frac{\pi}{4 H_{\mathrm{I}}^{2}}\left[1-\epsilon\left(\hat{R}_{-}^{\prime}\right) \frac{2}{\pi}\left(\cos ^{-1}\left(\frac{\hat{R}}{R_{\mathrm{o}}} \sqrt{1-H_{\mathrm{I}}^{2} R_{\mathrm{O}}^{2}}\right)\right)\left(\frac{R_{\mathrm{O}}^{2}-\hat{R}^{2}}{R_{\mathrm{O}}^{2}-\hat{R}^{2}\left(1-H_{\mathrm{I}}^{2} R_{\mathrm{O}}^{2}\right)}\right)^{3 / 2}\right] \\
& -\frac{\pi}{4 H_{\mathrm{O}}^{2}}\left[1-\epsilon\left(\hat{R}_{+}^{\prime}\right)\left(2+\frac{2}{\pi} \cos ^{-1}\left(\frac{\hat{R}}{R_{\mathrm{O}}} \sqrt{1-H_{\mathrm{O}}^{2} R_{\mathrm{O}}^{2}}\right)\right)\left(\frac{R_{\mathrm{O}}^{2}-\hat{R}^{2}}{R_{\mathrm{O}}^{2}-\hat{R}^{2}\left(1-H_{\mathrm{O}}^{2} R_{\mathrm{o}}^{2}\right)}\right)^{3 / 2}\right] \\
& +\frac{\hat{R}^{3}}{2 R_{\mathrm{O}}} \sqrt{R_{\mathrm{O}}^{2}-\hat{R}^{2}}\left[\frac{\left(H_{\mathrm{O}}^{2}-H_{\mathrm{I}}^{2}+T^{2}\right)}{\sqrt{1-c_{-}^{2} \hat{R}^{2}}}-\frac{\left(H_{\mathrm{O}}^{2}-H_{\mathrm{I}}^{2}-T^{2}\right)}{\sqrt{1-c_{+}^{2} \hat{R}^{2}}}\right] \\
& -\left[\frac{H_{\mathrm{O}}^{2}-H_{\mathrm{I}}^{2}+T^{2}}{4 T H_{\mathrm{I}}^{2}}-\frac{H_{\mathrm{O}}^{2}-H_{\mathrm{I}}^{2}-T^{2}}{4 T H_{\mathrm{O}}^{2}}\right] R_{\mathrm{o}} \sin ^{-1}\left(\frac{\hat{R}}{R_{\mathrm{o}}}\right) .
\end{aligned}
$$

The background action is obtained by setting $\hat{r}_{ \pm}=0$ in the above expressions (corresponding to having the complete dS space with Hubble parameter $H_{\mathrm{O}}$ ), giving us

$$
\bar{I}=-\frac{\pi}{2 G H_{\mathrm{O}}^{2}}\left[\left(1-H_{\mathrm{O}}^{2} a_{\mathrm{O}}^{2}\right)^{3 / 2}-1\right]
$$

which gives the Hartle-Hawking (under-the-barrier) wave function when substituted into Eq. (5.9) with $b=0$ and gives the Vilenkin version when $b=2 i a$.
Now as pointed out in [47] when the background geometry is a black hole there are two classically allowed (I and III) and one classically forbidden regions (II) as in the usual tunneling problem in quantum mechanics, as discussed for instance in Sec. III. Classically, the wall expands (or contracts) up to a classical turning point and then recollapses (or re-expands), but quantum mechanically it can tunnel under the barrier and resurface after the second turning point. In the $\mathrm{dS}$ to $\mathrm{dS}$ case however there is no region I $[15,16]$ and the situation as discussed in more 
detail in [16] is similar to tunneling from 'nothing'. In either case the WDW equation has two independent solutions as in Eq. (5.9) in each of these regions that need to be matched at the classical turning points. In the dS to dS case there is just the one turning point, the coefficients $a$ and $b$ will determine the two coefficients in region III through WKB matching conditions. In 'tunneling from nothing' discussions one usually imposes an additional boundary condition, either the outgoing wave condition of Vilenkin or the real wave function (coming from the so-called 'no-boundary' condition') of Hartle and Hawking. However, if no such condition is imposed, in general one of the two solutions in Eq. (5.9) will dominate in each region, depending on the sign of the real part of the action $I$.

The ratio

$$
\mathcal{P}(\mathcal{B} \rightarrow \mathcal{N})=\left|\frac{\Psi_{\mathcal{N}}}{\Psi_{\mathcal{B}}}\right|^{2}
$$

gives the probability of finding the system in the 'nucleated' state $\mathcal{N}$ versus being in the 'background' state $\mathcal{B}$, Notice that the two states $\mathcal{B}$ and $\mathcal{N}$ do not always have the meaning of 'initial' and 'final' states (see Sec. I): the transition can be clearly interpreted as happening in time if there is an initial classical motion of the bubble wall. In the cases in which there is no initial classical motion of the wall the interpretation is less clear. For this reason we will refer to the state $\mathcal{B}$ as the 'background' spacetime, instead of initial spacetime. Given that this is a relative probability, it does not have to be smaller than one, and we avoid the problem of the normalization of wave functionals.

The semiclassical wave function in the region III (i.e., the classical region where the brane spontaneously emerges from 'nothing') is obtained by analytically continuing the expression in Eq. (5.10). The denominator in Eq. (5.12) corresponds to the emergence from 'nothing' of dS space with the 'background' radius $H_{O}^{-1}$ and the relative probability is given by

$$
\mathcal{P}_{\text {out }}(\mathcal{B} \rightarrow \mathcal{N})=\frac{\left|\Psi_{\mathcal{N}}^{\text {out }}\right|^{2}}{\left|\Psi_{\mathcal{B}}^{\text {out }}\right|^{2}}=\frac{\left|a+i \frac{b}{2}\right|^{2} e^{2 \operatorname{Re}\left(I_{\text {out }}(\hat{R})\right)}+\left|a-i \frac{b}{2}\right|^{2} e^{-2 \operatorname{Re}\left(I_{\text {out }}(\hat{R})\right)}+2 \operatorname{Re}\left[\left(a+i \frac{b}{2}\right)\left(a^{*}-i \frac{b^{*}}{2}\right)\right] e^{2 i \operatorname{Im}\left(I_{\text {out }}(\hat{R})\right)}}{\left|a+i \frac{b}{2}\right|^{2} e^{2 \operatorname{Re}\left(I_{\text {out }}(0)\right)}+\left|a-i \frac{b}{2}\right|^{2} e^{-2 \operatorname{Re}\left(I_{\text {out }}(0)\right)}+2 \operatorname{Re}\left(\left(a+i \frac{b}{2}\right)\left(a^{*}-i \frac{b^{*}}{2}\right)\right) e^{2 i \operatorname{Im}\left(I_{\text {out }}(0)\right)}}
$$

As argued in [16] the dominant contribution comes from the ratio of the first terms in the numerator and denominator and gives precisely the expression that was obtained by the generalizations of CDL [5] and by Brown and Teitelboim [4], namely the expression in Eq. (4.25).

However it is important to emphasize that even though the final exponential term for the relative probability is the same as that obtained by the Euclidean instanton/diluteinstanton-gas method the expression before minimizing with respect to the wall radius i.e., Eq. (4.23) is very different from the (analytic continuation of) expression in Eq. (5.10). This suggests that the Lorenzian continuation of the CDL or BT Euclidean argument is strictly speaking just the minisuperspace calculation of Sec. IV B whilst the next in order of complication-namely the spherically $\left(S_{2}\right)$ symmetric Lorentzian calculation [16] based on [8] gives a completely different amplitude-even though the exponential term is in agreement. Furthermore as pointed out in Sec. III even in the flat space case the direct WKB calculation gives a different prefactor.

Finally, it is interesting to remark that in the Hamiltonian approach of $[7,8]$, in order to compare their results with the Euclidean approach of FGG [9], they describe a canonical Euclideanization of their approach by working on a static path and determine the relevant functions as $R, L$ in terms of the bubble location $\hat{R}$. Then they use $\hat{R}$ as the parameter that plays the role of Euclidean time in the Euclidean formalism. By doing this they successfully explain the Euclidean results of FGG in terms of a singular instanton that in the Hamiltonian approach corresponds to well behaved geometries. This Euclideanization essentially corresponds to the standard $t \rightarrow i t$ Wick rotation and does not correspond to the analytic continuations performed by CDL.

\section{B. Classical bubble trajectory after nucleation}

Now we want to determine the Penrose diagram for the trajectory of the bubble after nucleation.

The equations of motion for the wall are given by the junction conditions which are obtained by embedding the wall coordinates in dS spacetime. Assuming rotational invariance the metric of the wall is given by

$$
d s_{\Sigma}^{2}=-d \tau^{2}+R^{2}(\tau) d \Omega^{2} .
$$

We will first choose static coordinates for dS,

$$
d s^{2}=-\left(1-H^{2} r^{2}\right) d t^{2}+\frac{d r^{2}}{1-H^{2} r^{2}}+r^{2} d \Omega_{2}^{2},
$$

where $H^{-1}$ is the radius of dS. In these coordinates the wall radius is given by $r(\tau)=R(\tau)$. The junction conditions are

$$
\left(K^{+}\right)^{i}{ }_{j}-\left(K^{-}\right)_{j}^{i}=-4 \pi G \sigma \delta^{i}{ }_{j},
$$

where $K_{i j}^{ \pm}$is the extrinsic curvature at each side and $\sigma$ is the tension of the wall. In order to compute the extrinsic 
curvature it is helpful to use Gaussian normal coordinates in which $K_{a b}$ takes the simple form

$$
K_{a b}=-\Gamma_{a b}^{n}=-\frac{1}{2} \partial_{n} g_{a b}=-\frac{1}{2} n^{\mu} \partial_{\mu} g_{a b},
$$

where $n^{\mu}$ denotes the unit vector orthogonal to the wall and $g_{a b}$ is the induced metric at the wall. The whole computation then boils down to calculate the normal vector $n^{\mu}$ in an appropriate coordinate systems, from which we can compute the extrinsic curvature on the two sides of the wall and then enforce the junction conditions. To do so let us first denote the four-velocity of a point on the wall as $U^{\mu}$. In the static patch coordinate system, due to the spherical symmetry of the wall we have

$$
U_{S}^{\mu}=\left(\dot{t}_{d S}, \dot{R}, 0,0\right)
$$

where denotes the derivative with respect to proper time. Note that the normalization of the four-velocity $g_{\mu \nu} U_{S}^{\mu} U_{S}^{\nu}=-1$ implies the following relation between $\dot{t}_{d S}$ and $\dot{R}$ :

$$
\left(1-H^{2} R^{2}\right) \dot{t}_{d S}^{2}=1+\left(1-H^{2} R^{2}\right)^{-1} \dot{R}^{2} .
$$

To compute the normal vector to the wall first notice that this is orthogonal to the four-velocity $U^{\mu}$, i.e., $g_{\mu \nu} n^{\nu} U_{S}^{\mu}=0$. Using this condition and that $g_{\mu \nu} n^{\mu} n^{\nu}=1$ implies

$n^{\mu}=\left(\left(1-H^{2} R^{2}\right)^{-1} \dot{R}, \pm \sqrt{1-H^{2} R^{2}+\dot{R}^{2}}, 0,0\right)$.

One can now compute the junction condition. The $\theta$ component gives,

$$
\sqrt{1-H_{+}^{2} R^{2}+\dot{R}}-\sqrt{1-H_{-}^{2} R^{2}+\dot{R}^{2}}=4 \pi \sigma R,
$$

where the subscript \pm indicates the side of the wall where we are evaluating. After some manipulation this equation leads to Eq. (5.5), and so to a solution for the radius of the wall $R$. One also has the $\tau$ component of the extrinsic curvature, $K_{\tau \tau}=U^{\mu} U^{\nu} \nabla_{\nu} n_{\mu}=-n_{\mu} U^{\nu} \nabla_{\nu} U^{\mu}$, which can be interpreted as the normal acceleration of the wall. This implies that the trajectory followed by the wall is not a geodesic unless $K_{\tau \tau}$ vanishes. This can be evaluated in the static patch coordinates,

$$
K_{\tau \tau}=-\frac{\ddot{R}-H^{2} R}{\sqrt{1-H^{2} R^{2}+\dot{R}^{2}}}=-\frac{\sqrt{1-H^{2} R_{0}^{2}}}{R_{0}},
$$

where in the last step we have used Eq. (5.5). This last equation implies that the normal acceleration is a nonvanishing constant (since $R_{0} H<1$ ).

\section{Global slicing ${ }^{13}$}

We will now embed the wall in global coordinates, which are described in Eq. (A3). The metric is given by

$$
d s^{2}=\frac{1}{H^{2} \cos ^{2} T}\left(-d T^{2}+d \rho^{2}+\sin ^{2} \rho d \Omega^{2}\right),
$$

where $T$ is conformal time that varies from $-\pi / 2$ at $\mathcal{I}_{-}$ to $\pi / 2$ at $\mathcal{I}_{+}$. Embedding the wall metric in Eq. (5.14) into the global coordinates implies that $T(\tau)$ and $R(\tau)=$ $H^{-1} \sec T(\tau) \sin \rho(\tau)$. Also, plugging this back into Eq. (5.23) we have,

$$
-\dot{T}^{2}+\dot{\rho}^{2}=-H^{2} \cos ^{2} T
$$

where dots are derivatives with respect to proper time. Using this relation we find

$$
\dot{T}^{2}=\frac{H^{2} \cos ^{2} T}{1-\rho^{\prime 2}}, \quad \dot{\rho}^{2}=\frac{H^{2} \cos ^{2} T}{1-\rho^{\prime 2}} \rho^{\prime 2},
$$

with $\rho^{\prime} \equiv d \rho / d T$. To describe the trajectories of the wall in global coordinates we would like to find an expression for $\rho(T)$. Let us start by noticing that

$$
\begin{aligned}
\dot{R} & =\frac{1}{H} \frac{d T}{d \tau}\left(\tan T \sec T \sin \rho+\sec T \cos \rho \rho^{\prime}\right) \\
& =\frac{\tan T \sin \rho+\cos \rho \rho^{\prime}}{\sqrt{1-\rho^{\prime 2}}}=\sqrt{\frac{\sin ^{2} \rho}{H^{2} R_{0}^{2} \cos ^{2} T}-1}
\end{aligned}
$$

where we have used Eq. (5.25) and in the last step Eq. (5.5). The last equality is a first order nonlinear differential equation which determines the wall trajectory in conformal coordinates. The solution turns out to be remarkably simple; $\cos (\rho)=\sqrt{1-H^{2} R_{0}^{2}} \cos T$ as the reader may easily verify.

In practice it turns out that to obtain this solution it is more convenient to use Eq. (5.22). This is straightforward since it is also possible to write $\ddot{R}$, in terms of $\rho, T$ and derivatives of $\rho$ with respect to $T$. After substituting into Eq. (5.22) we get ${ }^{14}$

$$
K_{\tau \tau}=-\frac{\cos (T) \rho^{\prime \prime}-\sin (T) \rho^{\prime 3}+\sin (T) \rho^{\prime}}{\left(1-\rho^{\prime 2}\right)^{3 / 2}} H,
$$

given that $K_{\tau \tau}$ is constant this is a second order ordinary differential equation for $\rho$ as a function of $T$. Furthermore

\footnotetext{
${ }^{13}$ This part follows the last part of Appendix C of [47].

${ }^{14}$ Alternatively we can use that is possible to write $K_{\tau \tau}=-\frac{\dot{\beta}}{\dot{R}}$, with

$$
\beta \equiv \sqrt{1-H^{2} R^{2}+\dot{R}^{2}}=\frac{\cos \rho+\sin \rho \tan T \rho^{\prime}}{\sqrt{1-\rho^{\prime 2}}}
$$
}


this expression does not depend explicitly on $\rho$ and can be easily integrated if we rewrite it as

$$
\frac{\sqrt{1-H^{2} R_{0}^{2}}}{H R_{0}}=\cos ^{2}(T) \frac{d}{d T}\left(\frac{\sec T \rho^{\prime}}{\sqrt{1-\rho^{\prime 2}}}\right)
$$

which leads to

$$
\rho^{\prime}= \pm \frac{\sqrt{1-H^{2} R_{0}^{2}} \sin (T)}{\sqrt{H^{2} R_{0}^{2}+\left(1-H^{2} R_{0}^{2}\right) \sin ^{2}(T)}},
$$

where to fix one integration constant we have imposed $\rho^{\prime}=0$ at $T=0$, which comes from Eq. (5.5). We will keep the positive signs as it means that the wall speed increases. Notice that $\rho^{\prime}<1$ and that at $\mathcal{I}_{+}, \rho^{\prime}=\sqrt{1-H^{2} R_{0}^{2}}<1$. This expression can be integrated to obtain

$$
\cos (\rho)=\sqrt{1-H^{2} R_{0}^{2}} \cos T
$$

where we have used that at $T=0, R_{0}=\cos (\rho(0))$. Equation (5.30) determines the trajectory of the wall in global coordinates. Now let us analyze this expression, first note that this the trajectory never crosses the light cone $\rho=T$ since $T<\arccos \left(\sqrt{1-H R_{0}^{2}} \cos T\right)=\rho$. Also note that all trajectories end at $\rho=\pi / 2$, since at $T=\pi / 2$, $\cos \rho=0$. Moreover the world sheet of the trajectory is a timelike hyperboloid having $S O(3,1)$ invariance. To see this we can substitute Eq. (5.30) into the equations for the embedding of global dS from Eq. (A3). Hence the equation for the brane world volume in embedding coordinates is

$$
\begin{aligned}
X_{0} & =H^{-1} \tan T, \\
X_{1} & =H^{-1} \frac{\cos \rho}{\cos T}=H^{-1} \sqrt{1-H^{2} R_{0}^{2}}, \\
X_{2}^{2}+X_{3}^{2}+X_{4}^{2} & =R^{2}=\frac{\sin ^{2} \rho}{H^{2} \cos ^{2} T} \\
& =\frac{1}{H^{2} \cos ^{2} T}-\frac{1-H^{2} R_{0}^{2}}{H^{2}} .
\end{aligned}
$$

Hence we have the equation for the world sheet of the brane,

$$
-X_{0}^{2}+X_{2}^{2}+X_{3}^{2}+X_{4}^{2}=R_{0}^{2}
$$

This is the equation of a hyperboloid with $S O(3,1)$ symmetry, ${ }^{15}$ in other words it is three dimensional dS space

\footnotetext{
${ }^{15}$ In fact one could have guessed the solution simply by demanding that $X_{1}$ is a constant since that is the simplest choice for the embedding given that $R^{2}$ cannot be set to a constant, and then fixing the constant from the fact that at $T=0=X_{0}$, $R=R_{0}$.
}

with radius $R_{0}$ and corresponds to the Lorentzian rotation of Coleman's [3] Euclidean bounce solution.

In summary we have explicitly found a closed expression for the classical trajectory of the wall after nucleation given by Eq. (5.30). It corresponds to an $S O(3,1)$ symmetric hyperboloid. The speed is determined by Eq. (5.29) which even though it increases it never reaches the speed of light $\left(\left|\rho^{\prime}\right|<1\right)$. However it can easily be seen that if gravity is decoupled $(G \rightarrow 0)$ the turning point goes as $R_{0} \rightarrow 3 \sigma /\left(\Lambda_{O}-\Lambda_{I}\right)$ but $H \rightarrow 0$ and then $\rho^{\prime} \rightarrow 1$ reproducing the flat space results [48]. In general, the limiting speed differs from the speed of light by a small amount of order $M^{2} / M_{P}^{2}$ with $M$ the reference scale of the scalar field potential. Finally, from the Penrose Diagram in Fig. 9 it can be seen that the trajectory is such that a signal from the center of the bubble cannot reach the wall but in principle radiation from the wall can reach the observer at the center.

\section{Cosmological implications}

Let us now compare the cosmological differences between $\mathrm{CDL}$ and a closed universe after the tunneling transition. This revives the old question regarding the spatial curvature of the universe. After inflation this issue is considered less urgent since whatever the original curvature is, a short period of inflation is enough to render the universe essentially flat. However at least as a question of principle, especially

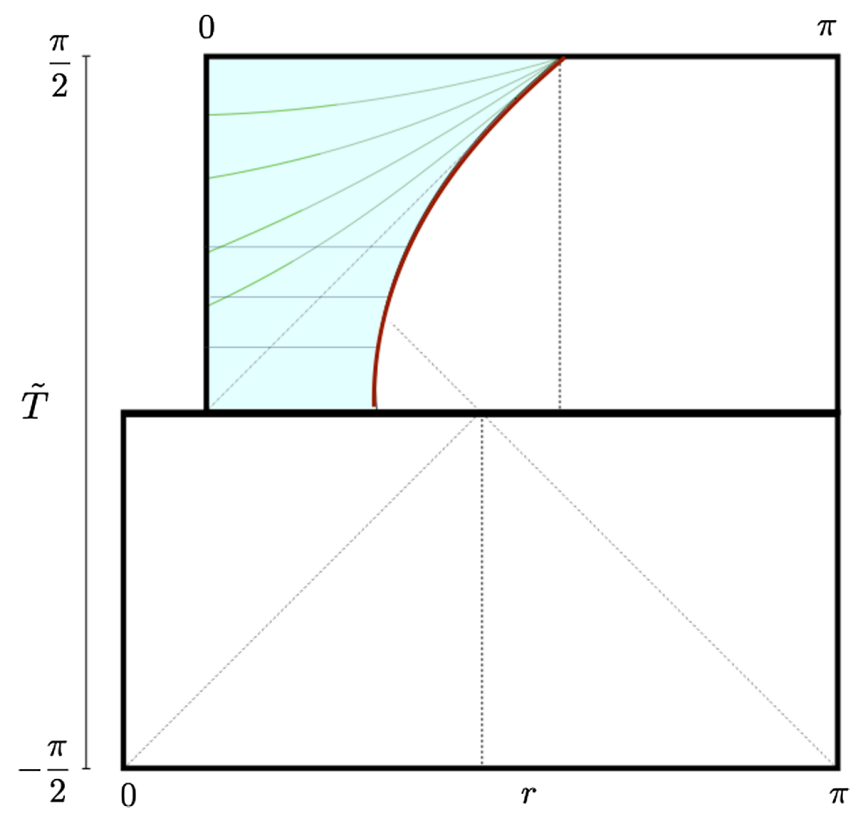

FIG. 9. Penrose diagram for the FMP dS to dS transition. The lower part is the universe before the transition. The upper part is the universe after the transition composed of two regions with different vacua separated by a wall, which is the red line. The equation of the wall is given by Eq. (5.30). The pale blue region is the part of the universe with the true vacuum, where the green dotted lines are open universe constant time slices and the blue dotted lines are closed universe constant time slices. 
concerning the question of whether the universe is infinite or finite (assuming that it does not have a nontrivial topology) and also potential observational effects, it is still relevant to address these differences. See for instance references [49-54] for a recent debate regarding current observations.

\section{Initial conditions and Inflation}

Vacuum decay offers a unique physical mechanism to provide the initial conditions for the evolution of the universe. The initial conditions for the classical cosmological evolution are the configurations of $\phi(t)$ and $a(t)$ right after tunneling that we will define as $t=0$.

(a) Open Universe: ${ }^{16}$ The Lorentzian equations of motion after CDL tunneling are those for an open $k=-1$ LFRW model with the standard cosmological evolution for a scalar field with canonical kinetic terms and potential $V(\phi)$,

$$
\begin{aligned}
\left(\frac{\dot{a}}{a}\right)^{2} & =\frac{8 \pi G}{3}\left(\frac{1}{2} \dot{\phi}^{2}+V(\phi)\right)+\frac{1}{a^{2}}, \\
0 & =\ddot{\phi}+3 H \dot{\phi}+V_{, \phi} .
\end{aligned}
$$

Initial conditions consistent with the smoothness of the CDL instanton are

$$
\dot{\phi}(0)=\phi(0)=0, \quad a(t)=t+\mathcal{O}\left(t^{3}\right) .
$$

Here $a(0)=0$ is a coordinate singularity. It is clear from Eqs. (5.34) that $\dot{a}$ could not be chosen to vanish at $t=0$ for positive potentials. We can see that initially the dynamics is dominated by the curvature term $1 / a^{2}$. The friction $(3 H)$ diverges at $t \rightarrow 0$, and then the initial $\dot{\phi}$ does not increase by much. From Eq. (5.34) we obtain the $\dot{H}$ equation for $k=-1$ :

$$
\dot{H}=\frac{\ddot{a}}{a}-\left(\frac{\dot{a}}{a}\right)^{2}=-4 \pi G \dot{\phi}^{2}-\frac{1}{a^{2}}<0,
$$

which guarantees in general that there is no local minimum value for $a(t)(\dot{a}=0 \Rightarrow \ddot{a}<0)$ and that there should be at least one point for which $a=0$ (the coordinate singularity). After a critical time of order $t^{*} \sim(\alpha V)^{-1 / 2}$ with $\alpha^{-1}=3 / 8 \pi G=3 M_{p}^{2}$, the potential starts to dominate, and the curvature term becomes less important as the universe expands. This could mark the onset of inflation as long as the slow roll conditions are satisfied. There are some conditions on the potential in order to achieve this scenario. In particular, it is required to have that $V^{\prime \prime} / V>1$ to be able to have a solution that satisfies the instanton boundary conditions [62] (see Fig. 10). This being the

\footnotetext{
${ }^{16}$ See $[10,55-61]$ for related discussions.
}

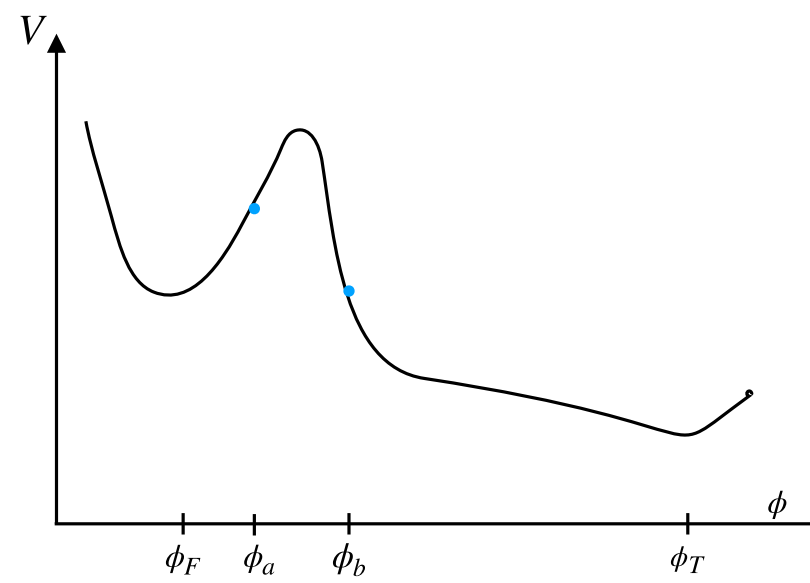

FIG. 10. The scalar field potential has a false vacuum at $\phi_{f}$ and an inflationary region on the right of $\phi_{T}$. In the standard CDL picture the field tunnels from $\phi_{a}$ to $\phi_{b}$.

opposite of the slow-roll condition, there has to be a curvature change in the scalar potential to eventually give rise to inflation. We may be more quantitative and follow the scale factor $a(t)$ and $\phi(t)$ for times smaller than $t^{*}$. In this case we can expand around the initial points $V(\phi(0)+\delta \phi) \sim \Lambda+\beta \delta \phi$ with $\beta=V^{\prime}(\phi(0))$ and find

$$
a(t)=\frac{\sinh (\lambda t)}{\lambda}, \quad \lambda^{2}=\alpha \Lambda=\frac{\Lambda}{3 M_{p}^{2}},
$$

and the scalar field

$$
\phi(t)=\frac{\beta}{3 \lambda^{2}}\left[\frac{\cosh (\lambda t)-1}{\cosh (\lambda t)+1}+\log \left(\frac{\cosh (\lambda t)+1}{2}\right)\right]
$$

From here we can explicitly verify that for the domain of validity of this regime $\left(t \leq t^{*}\right)$ the scale factor starts at zero and then increases linearly with time whereas the scalar field increases as $\phi(t) \lesssim \beta t^{2}$. For $t>t^{*} \equiv 1 / \sqrt{\lambda}, \Lambda$ dominates as long as $\beta$ is small enough $\left(\beta<\Lambda / M_{p}\right)$ such that $\Lambda$ dominates over both $\beta \phi$ and $\dot{\phi}^{2}$. In that case the universe starts a standard inflationary period, otherwise the field rolls fast and depending on the potential there may or may not be a standard period of inflation.

(b) Closed Universe. In the closed universe after tunneling the equations are $^{17}$

\footnotetext{
${ }^{17}$ For relatively recent discussions on the cosmology of closed universes see for instance [63-70].
} 


$$
\begin{aligned}
\left(\frac{\dot{a}}{a}\right)^{2} & =\frac{8 \pi G}{3}\left(\frac{1}{2} \dot{\phi}^{2}+V(\phi)\right)-\frac{1}{a^{2}}, \\
0 & =\ddot{\phi}+3 H \dot{\phi}+V_{, \phi} .
\end{aligned}
$$

The negative sign in the first equation due to the positive curvature $(k=1)$ changes the picture substantially. First note that the initial conditions can be fixed by imposing that at the turning point $\pi_{a}, \pi_{\phi}=0$. This implies that the right-hand side of the Friedmann equation above vanishes and the natural initial conditions can be [making a convenient choice for $\phi(0)]^{18}$

$$
\dot{\phi}(0)=\phi(0)=0, \quad \dot{a}(0)=0 .
$$

Contrary to the open case, there is no curvature dominated period since the curvature term has at best to balance the energy density term in order to have $(\dot{a} / a)^{2} \geq 0$. In particular at $t=0$ we have $a(0)=3 /(8 \pi G V(0)) \neq 0$. Different from the open universe case, the scale factor does not vanish. Also, the equation for $\dot{H}$ now reads

$$
\dot{H}=\frac{\ddot{a}}{a}-\left(\frac{\dot{a}}{a}\right)^{2}=-4 \pi G \dot{\phi}^{2}+\frac{1}{a^{2}},
$$

which, unlike the open and flat cases, can be positive or negative depending on the relative size of the kinetic terms and the curvature. Note that a positive curvature adds a positive contribution to the equation for $\dot{H}$. This means in particular that even though the universe is closed it does not necessarily recollapse [71]. Repeating the quantitative analysis as for the open case, assuming the energy density is dominated by $\Lambda$, we find

$$
a(t)=\frac{\cosh (\lambda t)}{\lambda}, \quad \lambda^{2}=\alpha \Lambda=\frac{\Lambda}{3 M_{p}^{2}} .
$$

Contrary to the open case the scale factor does not vanish at any point and after nucleation it starts with a minimum value of order $a(0) \geq a_{\min } \sim M_{p} / \sqrt{\Lambda}$ which is large enough to be in the regime for which the classical evolution equations are valid. The scalar field evolves as

\footnotetext{
${ }^{18}$ There are more general initial conditions for $G_{M N} \pi^{N} \pi^{M} \neq 0$ where $G^{M N}$ is the nonpositive metric in superspace. As we have described before (see Sec. IV D) when this is the case there also classical solutions that go from the false to the true vacua. In this case, the field oscillates around the true minima for a finite time until it reaches the points where $\dot{\phi}=\dot{a}=0$ that could be used as initial conditions for the rest of the evolution of the universe.
}

$\phi(t)=-\frac{\beta}{3 \lambda^{2}}\left[\log \cosh (\lambda t)-\operatorname{sech}^{2}(\lambda t)+1\right]$.

Now the Hubble parameter is $H=\dot{a} / a=\lambda \tanh (\lambda t)$, and

$\frac{\ddot{a}}{a}=\frac{8 \pi G}{3}\left(\Lambda-\dot{\phi}^{2}\right), \quad-\frac{\dot{H}}{H^{2}}=-\operatorname{csch}^{2}(\lambda t)$.

Unlike the open case there is no critical time before which the curvature term dominates over the $\Lambda$ contribution in the Friedmann equation. But in order to have the potential energy to be dominated by $\Lambda$ we need $\beta \phi<\Lambda$ which happens for times $t<$ $t^{*}\left(\Lambda / \beta M_{p}\right)^{2}$ and for having the kinetic energy suppressed with respect to the potential energy $\dot{\phi}^{2} \ll \Lambda$ which happens for times $t<t^{*}\left(\Lambda / \beta M_{p}\right)^{2}$ after that the universe stops accelerating. Both of these conditions are satisfied if $\beta \ll \Lambda / M_{p}$ which is the equivalent of slow-roll condition. So we have two different possible outcomes after nucleation depending on the value of $\beta$; a short period of relative fast roll and a few $e$-foldings before the standard slow-roll inflation or an inflationary period right after nucleation if $\beta \ll \Lambda / M_{p}$. The maximum number of $e$-foldings from this period would be

$$
\begin{aligned}
N_{\max } & =\int_{0}^{t_{c}} H d t=\log \cosh \left(\frac{t_{c}}{t^{*}}\right) \sim \frac{t_{c}}{t^{*}} \sim \frac{2}{\epsilon}, \\
t_{c} & =t^{*}\left(\frac{\Lambda}{\beta M_{p}}\right)^{2},
\end{aligned}
$$

where $\epsilon=M_{P}^{2} V_{, \phi}^{2} / 2 V^{2} \simeq M_{P}^{2} \beta^{2} / 2 \Lambda^{2}<1$ is the usual slow-roll parameter. This is the maximum number of $e$-foldings since at $t=0$ the nucleation happens at the minimum value of $a(t)$ by imposing $\dot{a}(0)=0$, implying $a(0)=a_{\min }=1 / \lambda$ [64]. Unlike the flat and open cases in which the initial value of $a$ at the start of inflation can be as small as possible, i.e., as small as the Planck scale $l_{P}$, in the closed case the existence of a lower bound for $a$ with $a_{\text {min }}$ much bigger than the Planck length implies a much stronger upper bound in the number of $e$-folds in order to fit with the present value size of the observable universe $a_{0}$ which could be estimated if $\delta=|\Omega-1|=1 / a_{0} H_{0}$ is measured; $N_{\max } \leq \ln a_{0} / a_{\min }$. Note that this is independent of the standard argument for $N \sim 60$ setting bounds on $\Omega$ today which if measured with enough precision may differentiate between open or closed universes.

\section{Density Perturbations}

The magnitude of density perturbations measured by $\delta \rho / \rho \sim H^{2} / \dot{\phi}$ grows from zero at $t=0$ to order 
$\delta \rho / \rho \sim \lambda^{3} / \beta$ close to $t=t_{c}$ which can be smaller or larger than standard slow-roll inflation depending on the values of $\beta$ and $\Lambda .^{19}$

If $\beta$ does not satisfy the slow-roll condition then $N=\int_{0}^{t^{*}} H d t=\log \cosh 1 \sim 1$. Therefore, it is clear that in this case the scalar potential will need to flatten up through an inflection point in order to have an adequate period of inflation afterwards. In both cases (slow or fast roll after bubble nucleation) the fact is that $k=1$ not only provides initial conditions for inflation but also contributes to the density perturbations since in general the presence of curvature provides a new scale. It affects the power spectrum in the sense that the long wavelength modes which exit the horizon during the early stages of inflation may carry imprints of the spatial curvature, whereas the short wavelength modes which exit the horizon later are not affected by the spatial curvature. This can also be seen if we compute the power spectrum for the inflationary perturbations. As it is well known the scalar field during inflation produces an adiabatic power spectrum of scalar and tensor perturbations. Whereas for flat universes the power spectrum is nearly scale invariant, this is not the case for open and closed universes. As the long wavelength modes leave the horizon carrying the imprint of the curvature they deviate from scale invariance at large scales. For small curvature $\Omega_{k}=k /\left(a_{0} H_{0}\right)<1$ the power spectrum can be written as ${ }^{20}[72,73]$

$$
\left.P(q)=A_{s} q^{\left(n_{s}-1\right)-3}\left(1-\frac{19}{8} \frac{k}{q^{2}}\right)+\mathcal{O}\left(\Omega_{k}^{2}\right)\right)
$$

where $A_{s}$ is the amplitude of the scalar fluctuations and $q$ is the comoving wave number. We have also neglected selfinteractions of the curvature perturbation. From Eq. (5.44) we can read that at large scales, or smaller $q$, the power spectrum is suppressed for a closed universe $(k=+1)$, but is enhanced for the same scales for an open universe. These deviations from scale invariance can have an effect on the CMB. At large scales the main contribution to the angular power spectrum $C_{l}$ is given by the Sachs-Wolfe effect ${ }^{21}$

$$
l(l+1) C_{l}=\frac{4 \pi}{25} A_{s}\left(1-\frac{19}{8} \frac{k r_{L}^{2}}{3(l-1)(l+2)}\right)
$$

\footnotetext{
${ }^{19}$ Note that in the open universe case, this quantity diverges at $t=0$ since $H$ diverges and $\dot{\phi}(0)=0$ there, and then increases with time.

${ }^{20} \mathrm{~A}$ more appropriate treatment of the power spectrum after tunneling needs to take into account the fluctuations of the wall. In the case of open inflation these translate into an excited initial state which also imply deviations from scale invariance [57]. For the closed universe solution obtained in Sec. IV B we leave the analysis of the inflationary perturbations for future work.

${ }^{21}$ For a derivation of the effect of the primordial power spectrum over Sachs-Wolfe effect see formula (2.6.19) of [74].
}

Where $r_{L}$ is the radial coordinate of the surface of last scattering. Then we see that at linear order in the curvature there is a suppression/enhancement of the low $l$ modes of the temperature anisotropy of the CMB depending on the sign of $k$. Note that this computation assumes that the only contribution from the curvature comes from inflation and, although this is not quite accurate, it works as a qualitative approximation. Another point is that the effect described is model independent but, as we mentioned before, there are other signatures that depend on each model that may have important consequences. For example, for the case of open inflation after CDL, there is a fast roll phase before slow roll, by which the authors of $[10,59]$ have argued that because of an anthropic bound on the duration of inflation the fast-roll phase translates into a potentially observable suppression of the low $l$ modes of the CMB. This implies that a negatively curved universe can have an effect which is indistinguishable from a positively curved universe, so in order to break this degeneracy it might be necessary to study higher order correlation functions of cosmological observables. At small scales it can be seen from Eq. (5.44) that the power spectra coincide. This means that the CMB power spectrum at large angles or small multipoles is suppressed with respect to the standard flat $\Lambda \mathrm{CDM}$ model. Since the effect through inflation is only present at large scales, the power spectrum coincides with the flat case for large multipoles $(\ell \gtrsim 30)$. This is the regime that has been tested most successfully, although recently several articles have found some evidence for the closed universe inflationary model from the latest CMB observations [50-52].

\section{Observational Implications and the String Landscape}

The implications of a closed universe after bubble nucleation may have important observational implications and would radically affect the dynamics of the string landscape. Let us list some of them.

(a) General Prediction. Due to the richness of the string landscape, it has been a serious challenge to identify concrete and general predictions that could be tested with the potential to rule out the landscape paradigm. The standard belief that bubble nucleation after vacuum decay gives rise only to an open universe, has been identified as the most concrete general prediction that could be subject to experimental test at some point. However, if the outcome is a closed universe, the prediction would be exactly the opposite. At the moment we cannot rule out the possibility that an open universe could also be allowed. Furthermore note that, in principle, the idea behind the landscape is that universes are continuously produced from a series of quantum tunneling among the many different vacua. If a parent universe is closed and a daughter universe is open, this chain of universe creation would not be possible. However if both parent and daughter universes are closed this is natural. Furthermore as 
emphasized before in this work, the string theory landscape is a result of brane nucleation rather than a CDL like process, and should be treated as in FMP.

(b) Bubble collisions. In the landscape picture, the continuous creation of inflationary bubbles may give rise to the possibility of bubble collisions that may have left some imprint in the CMB, gravitational waves, etc., (see for instance [75,76] for an overview). One important aspect of the treatment of bubble collisions is the symmetry after the collision. Assuming open universes, each bubble spatial section has an $\operatorname{SO}(3,1)$ symmetry which breaks to $S O(2,1)$ after the collision. Numerical relativity techniques have been developed during the past few years to address this problem (see for instance [77] and references therein). If the universe is closed the situation differs substantially, the finiteness of the volume of spatial sections affects the probability of collisions and the natural symmetry breaking would be from the $S O(4)$ symmetry of the corresponding three-spheres to $S O(3)$. This should modify the description of bubble collisions. A detailed discussion of this interesting effect lies beyond the scope of this article.

(c) Beyond bubbles and CDL. The natural outcome of a phase transition, such as vacuum decay, is through the nucleation of bubbles of the new vacuum. Bubbles are required if the universe has infinite volume as in flat and open spacetimes. If the spacetime is closed there is the possibility that the full space and not only a region within it can change to the new vacuum with nonvanishing probability since the volume is finite. Note also that, as we have seen in the previous section, the fact that in minisuperspace the kinetic energy for the scale factor is negative whereas that for the scalar field is positive [Eq. (4.11)], then the Hamiltonian constraint $\mathcal{H}=0$ allows to pass classically through the barrier of the scalar potential. This does not appear without gravity nor in the Euclidean approach nor in the Hartle-Hawking case without scalar fields. This would then be the leading contribution to the ratio of probabilities for the creation of each of the two dS spaces. Even though these conclusions may be artefacts of the minisuperspace approximation they would affect the structure of the landscape and deserve further study.

(d) Number of e-folds. For the open universe case, reference [10] extracted a lower bound on the number of $e$-folds during inflation $N>59.5$ similar to the observed bound $N>62$ (modulo logarithmic corrections due to the different epochs of matter/radiation domination after inflation). For $k=1$ as we discussed before, there is an upper bound on the number of $e$-folds (again with $\Delta N \sim 2 e$-folds with respect with the observed one) [66]. The reason being, as mentioned above, that for the closed universe, there is a minimum value of the scale factor $a_{\min } \sim M_{p} / \sqrt{\Lambda}$ that already starts large (contrary to the flat and open cases in which $a \sim 0$ before inflation and have in principle no limit on the maximum number of $e$-folds before reproducing the standard cosmology after inflation). This tends to favor concave models of inflation and disfavor models, such as chaotic inflation, that may have an essentially unlimited number of $e$-folds.

(e) Power suppression. As we have seen, both open and closed universes introduce a new cosmological scale, the curvature, and affect the density perturbations observed from the CMB. The net effect is a suppression of the power spectrum at large angles or small multipoles. This effect may have two different origins, one due to the curvature and the other if there is a period of fast rolling before inflation.

\section{CONCLUSIONS}

We have presented here an extension of the standard quantum mechanics WKB approximation to field theory and gravity. Expanding on previous approaches we developed a general geometric formalism to extend the WKB to wave functions in Wheeler's superspace and found explicit expressions for vacuum transition probabilities interpreted as ratios of the square of wave functions of the two different configurations for both field theory and gravity.

In field theory we presented explicitly two different cases with two vacua. The first is the standard scalar potential with two minima at finite field value and the second one with the second minimum corresponding to a runaway. In both cases we reproduce the standard Coleman results at leading order. In the second case we found the (potential) bound states and corresponding resonances to provide the explicit expression for the decay rate Eq. (3.24) which agrees with the Euclidean approach at leading order but differs in the prefactor. We further emphasized our approach is the natural generalization of the standard quantum mechanical WKB calculation and does not have subtle issues such as the handling of negative modes and trusting the dilute instanton approximation.

In the gravity case, our results can be summarized in two directions. In one way they confirm the results obtained by Euclidean methods. In particular we provide a Lorentzian perspective for the estimation and interpretation of the decay rates and the wall trajectory after tunneling, illustrating the validity of the Euclidean techniques at least as far as getting the leading exponential behavior goes. On the other hand we point out that there are substantial differences from the Euclidean approach. In particular for the minisuperspace case we found that:

(a) Selecting a very particular path we can reproduce exactly the decay rate as computed by CDL, however the interpretation of this path is not at all clear. 
(b) The natural transition rate gives simply the ratio of the two corresponding Hartle-Hawking wave functions, without a tension term as in Eq. (5.11) which gives dominant decay rate as compared to CDL. It seems then that this minisuperspace approach is actually a generalization of Hartle-Hawking/Vilenkin/Linde transitions than to CDL.

(c) The fact that the metric in superspace is not positive definite, as manifested for instance in the Hamiltonian constraint Eq. (4.11), allows for classical paths to connect the two dS vacua without the need to pass through or across the barrier. This will not have an exponentially suppressed decay rate but it depends on the initial conditions.

(d) The natural $\mathrm{dS}$ foliation corresponds to the global slicing which leads to a closed universe as in HartleHawking-Vilenkin-Linde unlike the open universe claimed by CDL. Here, recall that the driving argument was based on the analytic continuation triggered by the argument of the scalar field. Surfaces of constant $\phi$ would give rise to the hyperbolic foliation of spacetime. However, as noted already in [3] in the extreme thin-wall approximation we only have two values of the cosmological constant and there is no preferred dS space foliation. This means that already in the Euclidean framework described for instance in [4] horizontal slices of dS could have been chosen for the regions inside and outside the bubble with a closed universe.

(e) In minisuperspace there is no way to discuss bubble nucleation and therefore the transition should be interpreted between two entire dS spaces. This computation really makes sense only for $k=1$ since the spatial volume in global slicing is finite. A full description beyond minisuperspace is needed in order to properly include the bubble. In the Hamiltonian approach to quantum tunneling developed in $[7,8]$ it is clear that the geometry inside and outside the bubble is also of a closed universe [16]. In this approach the metric depends not only on time but also on the radial coordinate $r$. This reinforces our conclusion. This formalism only compares the transition between two different cosmological constants without considering a scalar field with the corresponding potential. This corresponds to the extreme thin-wall approximation or brane nucleation in string theory. A full Hamiltonian approach including the scalar field dynamics is not yet available.

(f) Our approach and that of $[7,8]$ started with a spherically symmetric geometry that was natural to describe the bubble after the transition but in principle we could have chosen a flat or negatively curved spacetime to start with. The fact that these spaces have infinite volume renders the volume integrals problematic. For instance in Eq. (4.8) the $2 \pi^{2}$ factors come from integrating the volume of the three-sphere which in the flat and open cases would diverge. This would require a proper volume regularization before extracting physical information. Similar volume integrals for closed slicings appear in the approach of $[7,8]$. Furthermore, in $[7,8]$ a prescription is provided to Euclideanize their results, the corresponding analytic continuation is simply $t \rightarrow i t$ which is not the analytic continuation proposed by CDL.

(g) We believe that the possibility that the geometry of the bubble after nucleation corresponds to a closed FLRW universe, contrary to CDL, should be seriously considered. Indeed this is the natural implication of brane nucleation in string theory as we have argued in this paper. This may have important physical implications if our universe is described in terms of a bubble after vacuum transitions as discussed at the end of the previous section. Besides the deep implications of having a finite against an infinite universe, with finite number of stars and galaxies, it may eventually be tested if there is a definite way of determining the curvature of the universe. For the string theory landscape, it will at least eliminate the standard claim that detecting a closed universe would rule out the multiverse. These are important cosmological questions that deserve further scrutiny.

\section{ACKNOWLEDGMENTS}

We thank Stefano Ansoldi, John Bohn, Cliff Burgess, Jim Hartle, Veronica Pasquarella, Jorge Santos, Andreas Schachner and Alexander Westphal for useful discussions. We also thank Andreas Schachner for comments on the manuscript. We thank Thomas Hertog for comments on a previous version. The work of S. C. is supported through MCIU (Spain) through Contract PGC2018-096646-A-I00 and by the IFT UAM- CSIC Centro de Excelencia Severo Ochoa SEV-2016-0597 Grant. F. M. is funded by a UKRI/ EPSRC Stephen Hawking fellowship, Grant Reference EP/ T017279/1 and partially supported by STFC consolidated Grant No. ST/P000681/1. The work of F. Q. has been partially supported by STFC consolidated Grants No. ST/ P000681/1 and No. ST/T000694/1.

\section{APPENDIX: dE SITTER GEOMETRY}

\section{Different foliations}

dS spacetime can be defined on a hypersurface,

$$
-X_{0}^{2}+X_{1}^{2}+X_{2}^{2}+X_{3}^{3}+X_{4}^{4}=\Lambda^{2},
$$

in five dimensional Minkowski

$$
d s^{2}=-d X_{0}^{2}+d X_{1}^{2}+d X_{2}^{2}+d X_{3}^{3}+d X_{4}^{4} .
$$




\section{a. Global slicing}

The entire dS space can be covered by

$$
\begin{aligned}
& X_{0}=\Lambda \sinh (t / \Lambda), \\
& X_{1}=\Lambda \cosh (t / \Lambda) \cos (\rho) \\
& X_{2}=\Lambda \cosh (t / \Lambda) \sin (\rho) \sin \theta \cos \phi, \\
& X_{3}=\Lambda \cosh (t / \Lambda) \sin (\rho) \sin \theta \sin \phi, \\
& X_{4}=\Lambda \cosh (t / \Lambda) \sin (\rho) \cos \theta,
\end{aligned}
$$

and the metric in Eq. (A2) becomes

$$
d s^{2}=-d t^{2}+\cosh ^{2}(t / \Lambda)\left(d \rho^{2}+\sin ^{2}(\rho) d \Omega_{2}^{2}\right),
$$

where constant time slices have $O(4)$ invariance. Unlike the other foliations this parametrization has the full $S O(4,1)$ invariance. The global conformal metric in Eq. (5.23) is related to this by the relation $\cosh (t / \Lambda)=1 / \cos (T)$ with $H \equiv 1 / \Lambda$.

Causal patch-The geometry as observed by an observer moving along a constant time hypersurface on one of the hemispheres, is given by the following foliation,

$$
\begin{aligned}
& X_{0}=\sqrt{\Lambda^{2}-r^{2}} \cosh (\tau / \Lambda), \\
& X_{1}=\sqrt{\Lambda^{2}-r^{2}} \sinh (\tau / \Lambda), \\
& X_{2}=r \sin \theta \cos \phi, \\
& X_{3}=r \sin \theta \sin \phi, \\
& X_{4}=r \cos \theta .
\end{aligned}
$$

The metric becomes

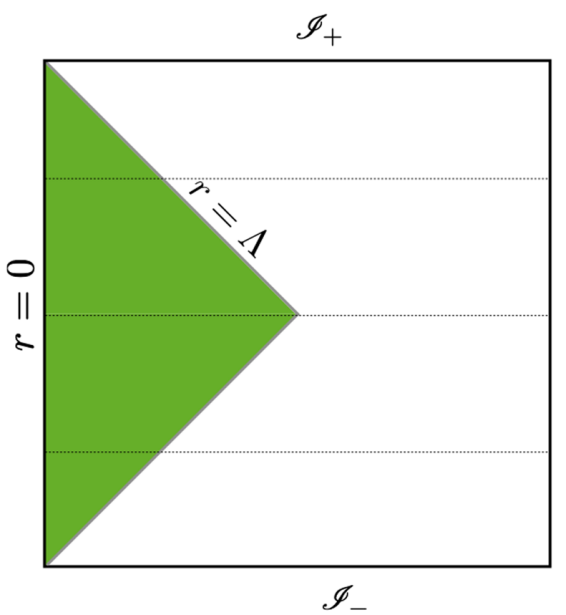

$d s^{2}=\left(1-\frac{r^{2}}{\Lambda^{2}}\right) d \tau^{2}+\left(1-\frac{r^{2}}{\Lambda^{2}}\right)^{-1} d \tau^{2}+r^{2} d \Omega_{2}^{2}$,

where $r$ varies between 0 and $\Lambda$ and $\tau$ goes from 0 to $\infty$. Notice that at $r=\Lambda$ the metric is not singular but there is a horizon.

Open slicings-Picking the coordinates,

$$
\begin{aligned}
& X_{0}=\Lambda \sin (\xi / \Lambda) \sinh (\chi), \\
& X_{1}=\Lambda \cos (\xi / \Lambda), \\
& X_{2}=\Lambda \sin (\xi / \Lambda) \cosh (\chi) \sin \theta \cos \phi, \\
& X_{3}=\Lambda \sin (\xi / \Lambda) \cosh (\chi) \sin \theta \sin \phi, \\
& X_{4}=\Lambda \sin (\xi / \Lambda) \cosh (\chi) \cos \theta,
\end{aligned}
$$

the metric in Eq. (A2) becomes,

$d s^{2}=d \xi^{2}+\Lambda^{2} \sin ^{2}(\xi / \Lambda)\left(-d \chi^{2}+\cosh ^{2} \chi^{2} d \Omega_{2}^{2}\right)$.

This foliation does not describe the full dS but only the causal patch of an observer at the center of the hyperboloid. The region covered is depicted in red in Fig. 11. Note that the analytical continuation $\chi \rightarrow i \chi$ leads to

$$
d s_{E}^{2}=d \xi^{2}+\Lambda^{2} \sin ^{2}(\xi / \Lambda)\left(d \chi^{2}+\cos ^{2} \chi^{2} d \Omega_{2}^{2}\right),
$$

which is the euclidean metric of a 4 sphere. This continuation was equivalent to make $X_{0} \rightarrow i X_{0}$. Another useful foliation is given by,

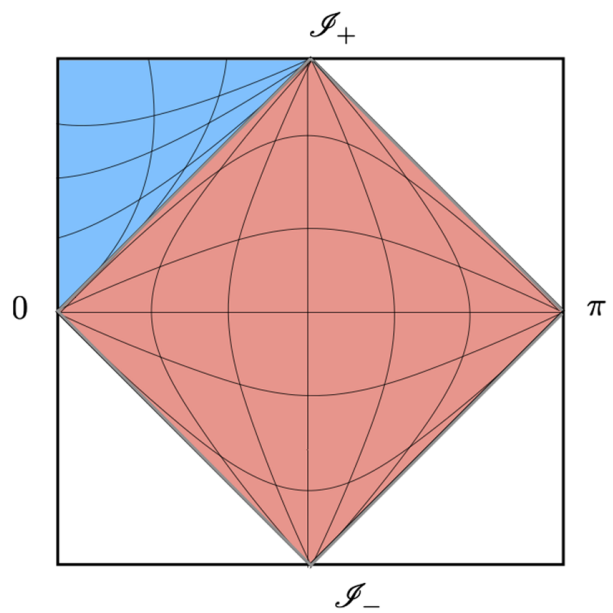

FIG. 11. Left panel: The green part is the region covered by the static patch of an observer at the south pole. The horizontal dotted lines are the constant time slices in the global coordinates. Right panel: Penrose diagram for the open slicing metrics in Eq. (A7) and Eq. (A10). The timelike lines are hypersurfaces of constant $\xi$ and spacelike lines are hypersurfaces of constant $\chi$. 


$$
\begin{aligned}
& X_{0}=\Lambda \cosh (\xi / \Lambda), \\
& X_{1}=\Lambda \sinh (\xi / \Lambda) \sinh (\chi), \\
& X_{2}=\Lambda \sinh (\xi / \Lambda) \cosh (\chi) \sin \theta \cos \phi, \\
& X_{3}=\Lambda \sinh (\xi / \Lambda) \cosh (\chi) \sin \theta \sin \phi, \\
& X_{4}=\Lambda \sinh (\xi / \Lambda) \cosh (\chi) \cos \theta,
\end{aligned}
$$

which foliates dS with spacelike hyperboloids. This foliation covers the region that lies between the outside region of the causal patch and the asymptotic future. It intersects the region covered by Eq. (A7), at $\xi=0$ which are the null surfaces for the observer on the hemisphere. The metric in Eq. (A2) becomes

$d s^{2}=-d \xi^{2}+\Lambda^{2} \sinh ^{2}(\xi / \Lambda)\left(d \chi^{2}+\sinh ^{2} \chi d \Omega_{2}^{2}\right)$,

which is also pictured as the region in blue in Fig. 11. To get the Euclidean continuation we need to do the transformations, $\chi \rightarrow i \chi$ and $\xi \rightarrow i \xi$, which lead to Eq. (A9). finally note that this transformation are equivalent to $X_{0} \rightarrow i X_{0}$.

\section{Analytic Continuation in CDL}

Given two vacua $\phi_{F}$ and $\phi_{T}$ such that $V\left(\phi_{F}\right)>$ $V\left(\phi_{T}\right) \geq 0$, according to [3] to calculate the scalar field that interpolates between the two minima first we need to consider the Euclidean solution that extremizes the action in order to derive the remaining geometry inside and outside the bubble. This was done in several steps:

(a) In field theory, the Euclidean bounce solution is $O(4)$ invariant in such a way that the scalar field depends on the Euclidean distance $\xi^{2}=|x|^{2}+\tau^{2}$. Analytic continuation changes this to a $O(3,1)$ and $\xi^{2} \rightarrow|x|^{2}-t^{2}$. Once gravity is included the corresponding line element is assumed to share that symmetry.

(b) Starting with the $O(4)$ symmetric Euclidean dS metric,

$$
d s^{2}=d \xi^{2}+\rho^{2}(\xi)\left(d \psi^{2}+\sin ^{2} \psi d \Omega_{2}^{2}\right)
$$

and writing $\psi \rightarrow \pi / 2+i \sigma$ we get the $O(3,1)$ invariant metric,

$$
d s^{2}=d \xi^{2}+\rho^{2}(\xi)\left(-d \sigma^{2}+\cosh ^{2} \sigma d \Omega_{2}^{2}\right)
$$

where $\sigma$ now runs from $-\infty$ to $\infty$. This spacetime is $\mathrm{dS}$ foliated by timelike hyperboloids. If we fix the angles and write $d \xi^{2}=\rho^{2}(\xi)\left(d y^{2}\right)$ we get

$$
d s^{2}=\rho^{2}(y)\left(-d \sigma^{2}+d y^{2}\right),
$$

whose geometry is represented by the causal diamond in Fig. 12. The wall lies within this region as indicated by the dotted timelike hypersurface in Fig. 12. An observer in the hemisphere will see the wall moving at a speed approaching the speed of light.

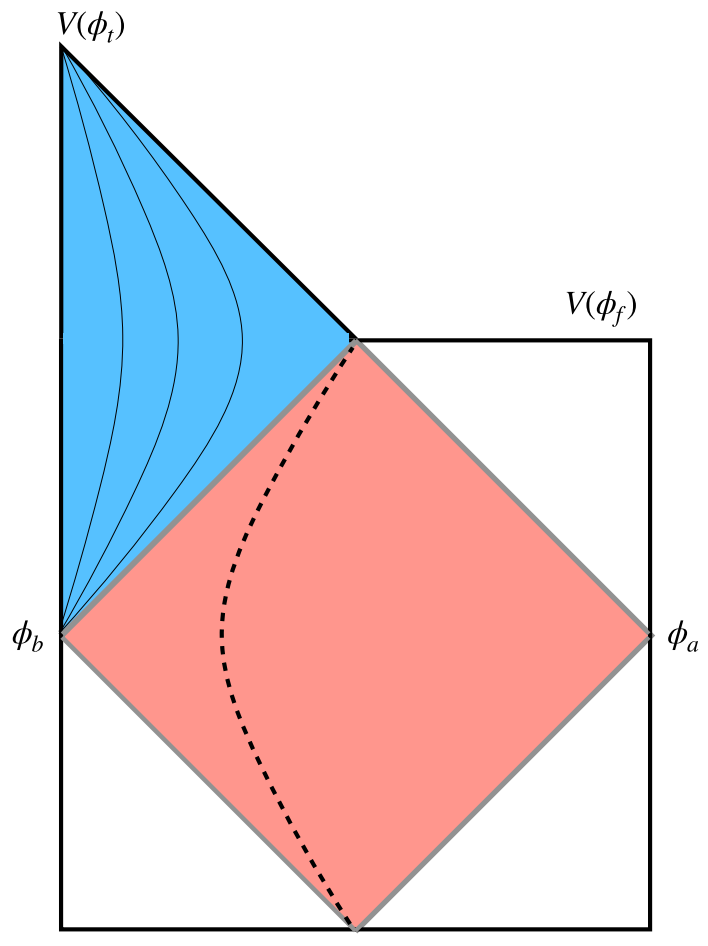

FIG. 12. Penrose diagram for the dS to Minkowski transition mediated by a $\mathrm{CdL}$ instanton. The red causal diagram is obtained after analytically continue the $O(4)$ instanton to $O(3,1)$ whose metric is given in Eq. (A14). The dotted line is the wall which is also a constant $\sigma$ slice. The blue region is open FRW with $V\left(\phi_{t}\right)=0$ obtained after analytically continue Eq. (A14) past the lightcone. In this region we also draw the constant radius hypersurfaces.

(c) This space is not geodesically complete, because for timelike geodesics it is possible to go past the lightlike hypersurfaces. To complete the description we can make a further analytical continuation $\sigma \rightarrow i \pi / 2+\chi$ and a rotation $\xi \rightarrow i t$ which leads to,

$d s^{2}=-d t^{2}+\rho^{2}(-i \xi)\left(d \chi^{2}+\sinh ^{2} \chi d \Omega_{2}^{2}\right)$.

This region describes an FRW open space, and covers the blue patch in Fig. 11. Note that the analytical continuation $\sigma \rightarrow-i \pi / 2+\chi$ describes the upper right region of the diagram. Constant time hypersurfaces end on $\mathcal{I}_{+}$at $\xi=\pi / 2 \mathrm{~d}$. To interpret the diagram in terms of dynamics of the scalar field, let us assume that the tunneling points where $\phi_{b}$ and $\phi_{a}$, as indicated in Fig. 10. Both points are hemispheres of $\mathrm{dS}$ where $\rho$ vanishes. The wall separating the two regions is inside the causal diamond as indicated in Fig. 12 by the dotted line. The left region outside the causal diamond describes the dynamics after the tunneling while the right region describes the false vacuum dynamics. Spacelike hypersurfaces in these region represent constant field surfaces. Then after the tunneling the field rolls down to the true vacuum $V\left(\phi_{t}\right)$ at $\mathcal{I}_{+}$. After analytic continuation the surfaces of constant field 
values correspond to constant values of $|x|^{2}-t^{2}$ which are hyperbolae. This defines the natural foliation of the spacetime.
In Fig. 12 we have assumed that the true vacuum is Minkowski. In the case of dS this only extends up to the horizontal line at $T=\pi / 2$.
[1] S. R. Coleman, The fate of the false vacuum. 1. Semiclassical theory, Phys. Rev. D 15, 2929 (1977).

[2] C. G. Callan, Jr. and S. R. Coleman, The fate of the false vacuum. 2. First quantum corrections, Phys. Rev. D 16, 1762 (1977).

[3] S. R. Coleman and F. De Luccia, Gravitational effects on and of vacuum decay, Phys. Rev. D 21, 3305 (1980).

[4] J. D. Brown and C. Teitelboim, Neutralization of the cosmological constant by membrane creation, Nucl. Phys. B297, 787 (1988).

[5] E. J. Weinberg, Classical Solutions in Quantum Field Theory: Solitons and Instantons in High Energy Physics, Cambridge Monographs on Mathematical Physics (Cambridge University Press, 2012), https://doi.org/ 10.1017/CBO9781139017787.

[6] A. Andreassen, D. Farhi, W. Frost, and M. D. Schwartz, Precision decay rate calculations in quantum field theory, Phys. Rev. D 95, 085011 (2017).

[7] W. Fischler, D. Morgan, and J. Polchinski, Quantum Nucleation of False Vacuum Bubbles, Phys. Rev. D 41, 2638 (1990).

[8] W. Fischler, D. Morgan, and J. Polchinski, Quantization of false vacuum bubbles: A Hamiltonian treatment of gravitational tunneling, Phys. Rev. D 42, 4042 (1990).

[9] E. Farhi, A. H. Guth, and J. Guven, Is it possible to create a universe in the laboratory by quantum tunneling?, Nucl. Phys. B339, 417 (1990).

[10] B. Freivogel, M. Kleban, M. Rodriguez Martinez, and L. Susskind, Observational consequences of a landscape, J. High Energy Phys. 03 (2006) 039.

[11] M. Kleban and M. Schillo, Spatial curvature falsifies eternal inflation, J. Cosmol. Astropart. Phys. 06 (2012) 029.

[12] J. Hartle and T. Hertog, Anthropic bounds on $\Lambda$ from the no-boundary quantum state, Phys. Rev. D 88, 123516 (2013).

[13] S. W. Hawking and T. Hertog, A smooth exit from eternal inflation?, J. High Energy Phys. 04 (2018) 147.

[14] B. Freivogel, V. E. Hubeny, A. Maloney, R. C. Myers, M. Rangamani, and S. Shenker, Inflation in AdS/CFT, J. High Energy Phys. 03 (2006) 007.

[15] T. C. Bachlechner, Inflation expels runaways, J. High Energy Phys. 12 (2016) 155.

[16] S. P. De Alwis, F. Muia, V. Pasquarella, and F. Quevedo, Quantum transitions between minkowski and de Sitter spacetimes, Fortschr. Phys. 68, 2000069 (2020).

[17] Z. Fu and D. Marolf, Bag-of-gold spacetimes, Euclidean wormholes, and inflation from domain walls in AdS/CFT, J. High Energy Phys. 11 (2019) 040.

[18] M. Mirbabayi, Uptunneling to de Sitter, J. High Energy Phys. 09 (2020) 070.
[19] R. Bousso and J. Polchinski, Quantization of four form fluxes and dynamical neutralization of the cosmological constant, J. High Energy Phys. 06 (2000) 006.

[20] A. Vilenkin, Creation of universes from nothing, Phys. Lett. 117B, 25 (1982).

[21] J. B. Hartle and S. W. Hawking, Wave function of the universe, Phys. Rev. D 28, 2960 (1983); Adv. Ser. Astrophys. Cosmol. 3, 174 (1987).

[22] A. Vilenkin, Quantum creation of universes, Phys. Rev. D 30, 509 (1984).

[23] A. D. Linde, Quantum creation of the inflationary universe, Lett. Nuovo Cimento 39, 401 (1984).

[24] R. Jinno and R. Sato, Negative mode problem of false vacuum decay revisited, arXiv:2010.04462.

[25] B. S. DeWitt, Quantum theory of gravity. 1. The canonical theory, Phys. Rev. 160, 1113 (1967).

[26] T. Banks, C. M. Bender, and T. T. Wu, Coupled anharmonic oscillators. 1. Equal mass case, Phys. Rev. D 8, 3346 (1973).

[27] T. Banks and C. M. Bender, Coupled anharmonic oscillators. ii. unequal-mass case, Phys. Rev. D 8, 3366 (1973).

[28] J. Gervais and B. Sakita, WKB wave function for systems with many degrees of freedom: A unified view of solitons and instantons, Phys. Rev. D 16, 3507 (1977).

[29] K. M. Bitar and S. Chang, Vacuum tunneling and fluctuations around a most probable escape path, Phys. Rev. D 18, 435 (1978).

[30] T. Tanaka, M. Sasaki, and K. Yamamoto, Field theoretic description of quantum fluctuations in multidimensional tunneling approach, Phys. Rev. D 49, 1039 (1994).

[31] S. W. Hawking, The quantum state of the universe, Adv. Ser. Astrophys. Cosmol. 3, 236 (1987).

[32] S. W. Hawking and D. N. Page, Operator ordering and the flatness of the universe, Adv. Ser. Astrophys. Cosmol. 3, 315 (1987).

[33] V. G. Lapchinsky and V. A. Rubakov, Canonical quantization of gravity and quantum field theory in curved spacetiME, Acta Phys. Pol. B 10, 1041 (1979).

[34] A. O. Barvinsky and C. Kiefer, Wheeler-DeWitt equation and Feynman diagrams, Nucl. Phys. B526, 509 (1998).

[35] A. Vilenkin, Boundary conditions in quantum cosmology, Phys. Rev. D 33, 3560 (1986).

[36] P. Hajicek, Elementary properties of a new kind of path integral, J. Math. Phys. (N.Y.) 27, 1800 (1986).

[37] H. D. Zeh, Time in quantum gravity, Phys. Lett. A 126, 311 (1988).

[38] J.H. Van Vleck, The correspondence principle in the statistical interpretation of quantum mechanics, Proc. Natl. Acad. Sci. U.S.A. 14, 178 (1928).

[39] L.S. Brown, Classical limit and the WKB approximation, Report No. RLO-1388-607. 
[40] E. Merzbacher, Quantum Mechanics, 3rd ed. (Wiley, New York, 1998), ISBN 0-471-88702-1.

[41] S. J. Parke, Gravity, the decay of the false vacuum and the new inflationary universe scenario, Phys. Lett. 121B, 313 (1983).

[42] J. Kristiano, R. D. Lambaga, and H. S. Ramadhan, Coleman-de Luccia tunneling wave function, Phys. Lett. B 796, 225 (2019).

[43] S. P. de Alwis, Wave function of the Universe and CMB fluctuations, Phys. Rev. D 100, 043544 (2019).

[44] J. J. Halliwell, J. B. Hartle, and T. Hertog, What is the noboundary wave function of the universe?, Phys. Rev. D 99, 043526 (2019).

[45] J. J. Blanco-Pillado, H. Deng, and A. Vilenkin, Flyover vacuum decay, J. Cosmol. Astropart. Phys. 12 (2019) 001.

[46] Ö. Güngör and G. D. Starkman, A classical, nonsingular, bouncing universe, J. Cosmol. Astropart. Phys. 04 (2021) 003.

[47] S. K. Blau, E. I. Guendelman, and A. H. Guth, The dynamics of false vacuum bubbles, Phys. Rev. D 35, 1747 (1987).

[48] A. Aguirre, M. C. Johnson, and M. Tysanner, Surviving the crash: Assessing the aftermath of cosmic bubble collisions, Phys. Rev. D 79, 123514 (2009).

[49] N. Aghanim (Planck Collaboration), Planck 2018 results. VI. Cosmological parameters, Astron. Astrophys. 641, A6 (2020).

[50] E. Di Valentino, A. Melchiorri, and J. Silk, Planck evidence for a closed Universe and a possible crisis for cosmology, Nat. Astron. 4, 196 (2020).

[51] W. Handley, Curvature tension: Evidence for a closed universe, Phys. Rev. D 103, L041301 (2021).

[52] G. Efstathiou and S. Gratton, The evidence for a spatially flat Universe, Mon. Not. R. Astron. Soc. 496, L91 (2020).

[53] E. Di Valentino, A. Melchiorri, and J. Silk, Cosmic discordance: Planck and luminosity distance data exclude LCDM, Astrophys. J. Lett. 908, L9 (2021).

[54] G. Efstathiou, Is the low CMB quadrupole a signature of spatial curvature?, Mon. Not. R. Astron. Soc. 343, L95 (2003).

[55] J. R. Gott, Creation of open universes from de Sitter space, Nature (London) 295, 304 (1982).

[56] B. Ratra and P. J. E. Peebles, Inflation in an open universe, Phys. Rev. D 52, 1837 (1995).

[57] M. Bucher, A. S. Goldhaber, and N. Turok, An open universe from inflation, Phys. Rev. D 52, 3314 (1995).

[58] A. D. Linde, M. Sasaki, and T. Tanaka, CMB in open inflation, Phys. Rev. D 59, 123522 (1999).

[59] R. Bousso, D. Harlow, and L. Senatore, Inflation after false vacuum decay: Observational prospects after Planck, Phys. Rev. D 91, 083527 (2015).
[60] B. Freivogel, M. Kleban, M. R. Martinez, and L. Susskind, Observational consequences of a landscape: Epilogue, arXiv:1404.2274.

[61] R. Bousso, D. Harlow, and L. Senatore, Inflation after false vacuum decay: New evidence from BICEP2, J. Cosmol. Astropart. Phys. 12 (2014) 019.

[62] L. G. Jensen and P. J. Steinhardt, Bubble nucleation and the Coleman-Weinberg model, Nucl. Phys. B237, 176 (1984).

[63] M. J. White and D. Scott, Why not consider closed universes?, Astrophys. J. 459, 415 (1996).

[64] G. Ellis, W. R. Stoeger, S. J. P. McEwan, and P. Dunsby, Dynamics of inflationary universes with positive spatial curvature, Gen. Relativ. Gravit. 34, 1445 (2002).

[65] A. D. Linde, Can we have inflation with Omega > 1?, J. Cosmol. Astropart. Phys. 05 (2003) 002.

[66] J. P. Uzan, U. Kirchner, and G. F. Ellis, WMAP data and the curvature of space, Mon. Not. R. Astron. Soc. 344, L65 (2003).

[67] A. Lasenby and C. Doran, Closed universes, de Sitter space and inflation, Phys. Rev. D 71, 063502 (2005).

[68] E. Masso, S. Mohanty, A. Nautiyal, and G. Zsembinszki, Imprint of spatial curvature on inflation power spectrum, Phys. Rev. D 78, 043534 (2008).

[69] B. Bonga, B. Gupt, and N. Yokomizo, Inflation in the closed FLRW model and the CMB, J. Cosmol. Astropart. Phys. 10 (2016) 031.

[70] B. Ratra, Inflation in a closed universe, Phys. Rev. D 96, 103534 (2017).

[71] J. D. Barrow, The premature recollapse problem in closed inflationary universes, Nucl. Phys. B296, 697 (1988).

[72] C. Cheung, P. Creminelli, A. L. Fitzpatrick, J. Kaplan, and 1. senatore, the effective field theory of inflation, J. High Energy Phys. 03 (2008) 014.

[73] P. Creminelli, A. Perko, L. Senatore, M. Simonović, and G. Trevisan, The physical squeezed limit: Consistency relations at order $q^{2}$, J. Cosmol. Astropart. Phys. 11 (2013) 015.

[74] S. Weinberg, Cosmology (Oxford University Press, New York, 2008), ISBN13 978-0-19-852682-7.

[75] M. Kleban, Cosmic bubble collisions, Classical Quantum Gravity 28, 204008 (2011).

[76] A. Aguirre and M.C. Johnson, A status report on the observability of cosmic bubble collisions, Rep. Prog. Phys. 74, 074901 (2011).

[77] M. C. Johnson, C. L. Wainwright, A. Aguirre, and H. V. Peiris, Simulating the Universe(s) III: Observables for the full bubble collision spacetime, J. Cosmol. Astropart. Phys. 07 (2016) 020. 